\title{
CONVERGENCE RATE OF THE TRUNCATED EULER- MARUYAMA METHOD FOR NEUTRAL STOCHASTIC DIFFERENTIAL DELAY EQUATIONS WITH MARKOVIAN SWITCHING*
}

\author{
Wei Zhang \\ School of Mathematical Sciences, Heilongjiang University, Harbin 150080, China \\ Email: weizhanghlj@163.com
}

\begin{abstract}
The key aim of this paper is to show the strong convergence of the truncated EulerMaruyama method for neutral stochastic differential delay equations (NSDDEs) with Markovian switching (MS) without the linear growth condition. We present the truncated Euler-Maruyama method of NSDDEs-MS and consider its moment boundedness under the local Lipschitz condition plus Khasminskii-type condition. We also study its strong convergence rates at time $T$ and over a finite interval $[0, T]$. Some numerical examples are given to illustrate the theoretical results.
\end{abstract}

Mathematics subject classification: 65L20, 65C40.

Key words: Neutral stochastic differential delay equations, Truncated Euler-Maruyama method, Local Lipschitz condition, Khasminskii-type condition, Markovian switching.

\section{Introduction}

Due to better explanations of the phenomena, stochastic differential equations (SDEs) and stochastic differential delay equations (SDDEs) which are more efficient and reliable models in dynamical systems receive more and more attention recently. Systems with Markovian switching which were introduced by Kac and Krasovskii (see in [6]) have become a powerful tool in the modelling of practical systems (see in $[7,21]$ and the references therein). The structures and parameters of the underlying neutral stochastic differential delay equations (NSDDEs) may change abruptly because of environmental disturbances etc. We use the continuous-time Markovian chain $r(t)$ to model such abrupt changes. Thus, the underlying NSDDE

$$
d[x(t)-D(x(t-\tau))]=F(x(t), x(t-\tau)) d t+G(x(t), x(t-\tau)) d w(t)
$$

becomes the following NSDDE with Markovian switching (MS), abbreviated as NSDDE-MS

$$
d[x(t)-D(x(t-\tau), r(t))]=F(x(t), x(t-\tau), r(t)) d t+G(x(t), x(t-\tau), r(t)) d w(t) .
$$

Since most SDEs-MS and SDDEs-MS have no explicit solutions, numerical methods to approximate the underlying solutions become a useful technique. There are many results concerned with the numerical solutions of SDEs-MS and SDDEs-MS in recent years (see e.g., $[11,13,15,19,20,25-27,31])$. Up to now, most of the strong convergence theories are considered under the global Lipschitz condition or the local Lipschitz condition and the linear growth

\footnotetext{
* Received October 23, 2018 / Revised version received March 3, 2019 / Accepted June 20, 2019 /

Published online August 13, 2019 /
} 
condition. In 2003, Kolmanovskii et al. [8] discussed some important properties of the solutions e.g. boundedness and stability for NSDDEs-MS under Global Lipschitz and linear growth condition. Some numerical solutions to NSDDEs-MS were also discussed in [23, 24, 30]. However, lots of NSDDEs-MS do not satisfy the linear growth condition and there are a few results about NSDDEs-MS without linear growth condition (see, e.g., [9,10,22] and the references cited therein). As we know, the efficient explicit Euler schemes of SDEs (even without Markovian switching) can not be convergent in strong sense with the super-linearly growing drift coefficients, see for example [4]. In 2012, Hutzethaler et al. presented tamed Euler method which can solve this problem [5]. In [20], Nguyen et al. proposed tamed-Euler method for hybrid stochastic differential equations with Markovian switching. In 2015 and 2016, Mao [17, 18] presented the truncated Euler-Maruyama (EM) method for SDEs and gave its convergence rate. The truncated (EM) method has been discussed intensively by some authors (see, e.g., [1,3, 12]). Recently, we established the strong convergence theory of the partially truncated EM method for a class of SDDEs [28], and considered convergence rates of the truncated EM method for stochastic functional differential equations (SFDEs) in [29] under the local Lipschitz condition plus the Khasminskii-type condition. Though there are many papers on numerical methods for NSDDEs-MS. Up to now, there is little numerical theory on NSDDEs-MS under local Lipschitz condition and Khasminskii-type condition. Obviously, NSDDEs-MS is a generalization of SDEs and SDDEs. However, some new difficulties will appear due to the nonlinear growth condition.

Owning to cheap computational cost, explicit numerical methods are indeed to be discussed. Our aim here is to develop the truncated EM method of NSDDEs-MS under local Lipschitz condition and Khasminskii-type condition. For the convenience, we will, in Section 2, develop the truncated EM method. We will study the moment boundedness in Section 3 and the convergence rate of the truncated EM method in Section 4, respectively. Some numerical examples are given in Section 5. The conclusion of our paper is presented in Section 6 .

\section{The Truncated Euler-Maruyama Method}

Throughout this paper, assume that $\left(\Omega, \mathcal{F},\left\{\mathcal{F}_{t}\right\}_{t \geq 0}, \mathbb{P}\right)$ denotes a complete probability space with a filtration $\left\{\mathcal{F}_{t}\right\}_{t \geq 0}$ satisfying the usual conditions (i.e, it is right continuous and increasing while $\mathcal{F}_{0}$ contains all $\mathbb{P}$-null sets). Let $\mathbb{E}$ be the expectation corresponding to $\mathbb{P}$. Assume that $w(t)=\left(w_{1}(t), w_{2}(t), \cdots, w_{m}(t)\right)^{T}$ is an m-dimensional Brownian motion defined on the probability space. Let $|\cdot|$ denote both Euclidean norm in $\mathbb{R}^{n}$ and the trace norm in $\mathbb{R}^{n \times m}$. Denote by $C\left([-\tau, 0], \mathbb{R}^{n}\right)$ the family of continuous functions from $[-\tau, 0]$ to $\mathbb{R}^{n}$ with the norm $\|\varphi\|=\sup _{-\tau \leq u \leq 0}|\varphi(u)|$. For $a, b \in \mathbb{R}$, we use $a \vee b$ and $a \wedge b$ for $\max \{a, b\}$ and $\min \{a, b\}$, respectively. If $D$ is a set of $\Omega$, its indicator function is denoted by $\mathbf{1}_{D} \cdot\lfloor x\rfloor$ denotes the biggest integer which is not bigger than $x$.

Let $r(t)(t \geq 0)$ be a right-continuous Markovian chain on the probability space $\left(\Omega, \mathcal{F},\left\{\mathcal{F}_{t}\right\}_{t \geq 0}\right.$, $\mathbb{P})$, taking values in a finite state space $S=\{1,2, \cdots, Q\}$ with $\Gamma=\left(\gamma_{i j}\right)_{Q \times Q}$ given by

$$
\mathbb{P}\{(r(t+\Delta))=j \mid r(t)=i\}= \begin{cases}\gamma_{i j} \Delta+o(\Delta), & \text { if } i \neq j \\ 1+\gamma_{i j} \Delta+o(\Delta), & \text { if } i=j\end{cases}
$$

where $\Delta>0$. Here $\gamma_{i j} \geq 0$ is the transition from $i$ to $j$ if $i \neq j$, while $\gamma_{i i}=-\sum_{j \neq i} \gamma_{i j}$.

Assume that the Markovian chain $r(t)$ is independent of the Brownian motion $w(t)$. It is known that almost every sample path of $r(t)$ is a right-continuous step function with a finite 
number of simple jumps in any finite subinterval of $\mathbb{R}_{+}$. In other words, there is a sequence of stopping times $0=\tau_{0}<\tau_{1}<\cdots<\tau_{k} \rightarrow \infty$ almost surely such that

$$
r(t)=\sum_{k=0}^{\infty} r\left(\tau_{k}\right) \mathbf{1}_{\left[\tau_{k}, \tau_{k+1}\right)}(t),
$$

$r(t)$ is a constant on every interval $\left[\tau_{k}, \tau_{k+1}\right)$, for every $k \geq 0$

$$
r(t)=r\left(\tau_{k}\right), \text { on } \tau_{k} \leq t<\tau_{k+1} .
$$

Consider an $n$-dimensional NSDDE-MS

$$
d[x(t)-D(x(t-\tau), r(t))]=F(x(t), x(t-\tau), r(t)) d t+G(x(t), x(t-\tau), r(t)) d w(t)
$$

for $t \geq 0$ with initial data $x_{0}=\xi \in C\left([-\tau, 0] ; \mathbb{R}^{n}\right)$ and $r(0)=r_{0}$. Here $D: \mathbb{R}^{n} \times S \longrightarrow \mathbb{R}^{n}$, $f: \mathbb{R}^{n} \times \mathbb{R}^{n} \times S \longrightarrow \mathbb{R}^{n}$ and $g: \mathbb{R}^{n} \times \mathbb{R}^{n} \times S \longrightarrow \mathbb{R}^{n \times m}$.

We impose the following four assumptions as hypotheses:

(A1) For each integer $R \geq 1$, there exists a positive constant $C_{R}$ such that

$$
|F(x, y, i)-F(\bar{x}, \bar{y}, i)| \vee|G(x, y, i)-G(\bar{x}, \bar{y}, i)| \leq C_{R}(|x-\bar{x}|+|y-\bar{y}|)
$$

for all $x, y \in \mathbb{R}^{n}$ and $i \in S$ with $|x| \vee|y| \vee|\bar{x}| \vee|\bar{y}| \leq R$.

(A2) There is a pair of constants $p>2$ and $K_{1}>0$ such that

$$
(x-D(y, i))^{T} F(x, y, i)+\frac{p-1}{2}|G(x, y, i)|^{2} \leq K_{1}\left(1+|x|^{2}+|y|^{2}\right)
$$

for all $x, y \in \mathbb{R}^{n}$ and $i \in S$.

(A3) Assume that $D(0, i)=0$ and there exists a positive $\kappa \in(0,1)$ such that

$$
|D(x, i)-D(y, i)| \leq \kappa|x-y|
$$

for every $x, y \in \mathbb{R}^{n}$ and $i \in S$.

From (2.5), we can see that

$$
|D(x, i)| \leq \kappa|x|
$$

(A4) There is a constant $K_{0}>0$ such that

$$
|\xi(t)-\xi(s)| \leq K_{0}|t-s|^{1 / 2}
$$

for all $s, t \in[-\tau, 0]$.

It is easy to show the following lemma by the similar way of Theorem 3.1 in [14] and Theorem 2.4 in $[16]$.

Lemma 2.1. Under $(A 1)-(A 3)$, the $N S D D E-M S(2.2)$ has a unique global solution $x(t)$ and, moreover

$$
\sup _{t \in[-\tau, T]} \mathbb{E}|x(t)|^{p} \leq C
$$

for all $T>0$, where $C$ stands for a generic positive real constant (but independent of $\Delta$ and $R$ later) and its value may change between occurrences. 
In order to define the truncated Euler-Maruyama mthod, we first choose a strictly increasing continuous function $\phi: \mathbb{R}_{+} \rightarrow \mathbb{R}_{+}$such that $\phi(v) \rightarrow \infty$ as $v \rightarrow \infty$ and

$$
\sup _{|x| \vee|y| \leq v}(|F(x, y, i)| \vee|G(x, y, i)|) \leq \phi(v), \quad \forall v \geq 0 .
$$

$\phi^{-1}$ is the inverse function of $\phi$ and a strictly increasing continuous function from $[\phi(0), \infty)$ to $\mathbb{R}_{+}$. Then we can choose a number $\Delta^{*} \in(0,1]$ and a strictly decreasing function $\psi:\left(0, \Delta^{*}\right] \rightarrow$ $(0, \infty)$ such that

$$
\begin{aligned}
& \psi\left(\Delta^{*}\right) \geq \phi(2), \\
& \lim _{\Delta \rightarrow 0} \psi(\Delta)=\infty, \\
& \Delta^{1 / 4} \psi(\Delta) \leq 1, \quad \forall \Delta \in(0,1) .
\end{aligned}
$$

For a given step size $\Delta \in(0,1)$, we define the truncated functions

$$
\begin{aligned}
& F_{\Delta}(x, y, i)=F\left(\left(|x| \wedge \phi^{-1}(\psi(\Delta))\right) \frac{x}{|x|},\left(|y| \wedge \phi^{-1}(\psi(\Delta))\right) \frac{y}{|y|}, i\right) \\
& G_{\Delta}(x, y, i)=G\left(\left(|x| \wedge \phi^{-1}(\psi(\Delta))\right) \frac{x}{|x|},\left(|y| \wedge \phi^{-1}(\psi(\Delta))\right) \frac{y}{|y|}, i\right)
\end{aligned}
$$

for all $x, y \in \mathbb{R}^{n}$ and $i \in S$, where we set $\frac{x}{|x|}=0$ when $x=0$. It is obvious that

$$
\left|F_{\Delta}(x, y, i)\right| \vee\left|G_{\Delta}(x, y, i)\right| \leq \phi\left(\phi^{-1}(\psi(\Delta))\right)=\psi(\Delta) \quad \forall x, y \in \mathbb{R}^{n} .
$$

That is, although both $F$ and $G$ may be unbounded, both truncated functions $F_{\Delta}$ and $G_{\Delta}$ are bounded.

We also impose the following assumption:

(H1) There exists a pair of constant $p>2$ and $K_{3}>0$ such that

$$
(x-D(y, i))^{T} F_{\Delta}(x, y, i)+\frac{p-1}{2}\left|G_{\Delta}(x, y, i)\right|^{2} \leq K_{3}\left(1+|x|^{2}+|y|^{2}\right)
$$

for all $\Delta \in\left(0, \Delta^{*}\right], x, y \in \mathbb{R}^{n}$ and $i \in S$.

Lemma 2.2. Let $r_{k}^{\Delta}=r(k \Delta)$ for $\Delta>0$ and $k=0,1,2, \cdots$, then $\left\{r_{k}^{\Delta}, k=0,1,2, \cdots\right\}$ is discrete Markovian chain with the one-step transition probability matrix

$$
\mathbb{P}(\Delta)=\left(\mathbb{P}_{i j}(\Delta)\right)_{Q \times Q}=e^{\Delta \Gamma} .
$$

Since the $\gamma_{i j}$ are independent of $x$, before computing $x$ the paths of $r$ can be generated independently of $x$.

From now on, we will consider NSDDE-MS (2.2). Let the step size $\Delta \in(0,1)$. Without loss of any generality, we may assume that $\tau / \Delta=N$ where $N \in \mathbb{N}$. We simulate the discrete Markovian chain $\left\{r_{k}^{\Delta}, k=0,1,2, \cdots\right\}$ as follows: Compute the one-step transition probability matrix $\mathbb{P}(\Delta)$. Let $r_{0}^{\Delta}=i_{0}$ and generate a random number $\eta_{1}$, which is uniformly distributed in $[0,1]$. Define

$$
r_{1}^{\Delta}= \begin{cases}i_{1}, \quad \text { if } \quad i_{1} \in S-\{Q\} \text { such that } \sum_{j=1}^{i_{1}-1} \mathbb{P}_{i_{0} j}(\Delta) \leq \eta_{1}<\sum_{j=1}^{i_{1}} \mathbb{P}_{i_{0} j}(\Delta), \\ Q, \quad \text { if } \sum_{j=1}^{Q-1} \mathbb{P}_{i_{0} j}(\Delta) \leq \eta_{1},\end{cases}
$$


where $\sum_{j=1}^{0} \mathbb{P}_{i_{0} j}(\Delta)=: 0$. Generate independently a new random number $\eta_{2}$ which is uniformly distributed in $[0,1]$ and we define

$$
r_{2}^{\Delta}=\left\{\begin{array}{l}
i_{2}, \quad \text { if } i_{2} \in S-\{Q\} \text { such that } \sum_{j=1}^{i_{2}-1} \mathbb{P}_{i_{1} j}(\Delta) \leq \eta_{2}<\sum_{j=1}^{i_{2}} \mathbb{P}_{i_{1} j}(\Delta), \\
Q, \quad \text { if } \sum_{j=1}^{Q-1} \mathbb{P}_{i_{1} j}(\Delta) \leq \eta_{2} .
\end{array}\right.
$$

Repeating this procedure, the trajectory $\left\{r_{k}^{\Delta}, k=0,1,2, \cdots\right\}$ can be generated. The procedure can be carried out independently to obtain more trajectories. Now we can form the discretetime truncated EM numerical solutions $Y_{\Delta}\left(t_{k}\right)=x\left(t_{k}\right)$ by setting $Y_{\Delta}(0)=x(0), r_{0}^{\Delta}=i_{0}$ and performing

$$
\left\{\begin{aligned}
Y_{\Delta}\left(t_{k}\right)=\xi\left(t_{k}\right), \quad & k=-N,-N+1, \cdots, 0, \\
Y_{\Delta}\left(t_{k+1}\right)=Y_{\Delta}\left(t_{k}\right) & +D\left(Y_{\Delta}\left(t_{k+1-N}\right), r_{k+1}^{\Delta}\right)-D\left(Y_{\Delta}\left(t_{k-N}\right), r_{k}^{\Delta}\right)+F_{\Delta}\left(Y_{\Delta}\left(t_{k}\right), Y_{\Delta}\left(t_{k-N}\right), r_{k}^{\Delta}\right) \\
& +G_{\Delta}\left(Y_{\Delta}\left(t_{k}\right), Y_{\Delta}\left(t_{k-N}\right), r_{k}^{\Delta}\right) \Delta w_{k}, \quad k=0,1,2, \cdots,
\end{aligned}\right.
$$

where $w_{k}=w\left(t_{k}\right)$ and $\Delta w_{k}=w_{k+1}-w_{k}$. Define $\bar{r}(t)=r_{k}^{\Delta}=r\left(t_{k}\right)$ for $t \in\left[t_{k}, t_{k+1}\right)$. Let us now establish the continuous-time truncated EM solutions. The first one is defined by

$$
\bar{y}_{\Delta}(t)=\sum_{k=-N}^{\infty} Y_{\Delta}\left(t_{k}\right) \mathbf{1}_{\left[t_{k}, t_{k+1}\right)}(t) .
$$

This is not a continuous simple step process. Let us refer this as the continuous-time stepprocess truncated EM solution. The other one is defined by

$$
\left\{\begin{aligned}
y_{\Delta}(t)=\xi(t), & \quad t \in[-\tau, 0] \\
y_{\Delta}(t)=\xi(0) & +D\left(\bar{y}_{\Delta}(t-\tau), \bar{r}(t)\right)-D\left(\xi(-\tau), r_{0}^{\Delta}\right)+\int_{0}^{t} F_{\Delta}\left(\bar{y}_{\Delta}(s), \bar{y}_{\Delta}(s-\tau), \bar{r}(s)\right) d s \\
& +\int_{0}^{t} G_{\Delta}\left(\bar{y}_{\Delta}(s), \bar{y}_{\Delta}(s-\tau), \bar{r}(s)\right) d w(s) .
\end{aligned}\right.
$$

Clearly, (2.17) can also be written as

$$
y_{\Delta}(t)=Y_{\Delta}\left(t_{k}\right)+\int_{t_{k}}^{t} F_{\Delta}\left(\bar{y}_{\Delta}(s), \bar{y}_{\Delta}(s-\tau), \bar{r}(s)\right) d s+\int_{t_{k}}^{t} G_{\Delta}\left(\bar{y}_{\Delta}(s), \bar{y}_{\Delta}(s-\tau), \bar{r}(s)\right) d w(s) .
$$

We will refer this as the continuous-time continuous-sample truncated EM solution. In particular, this shows that $Y_{\Delta}\left(t_{k}\right)=\bar{y}_{\Delta}\left(t_{k}\right)=y_{\Delta}\left(t_{k}\right)$ for all $k=0,1, \cdots$.

Moreover, for all $t \in[0, T]$, we have

$$
\begin{aligned}
\mathbb{E}\left|\bar{y}_{\Delta}(t)\right| & \leq \sup _{0 \leq s \leq t} \mathbb{E}\left|\bar{y}_{\Delta}(s)\right|=\sup _{0 \leq t_{k} \leq t} \mathbb{E}\left|Y_{\Delta}\left(t_{k}\right)\right| \\
& =\sup _{0 \leq t_{k} \leq t} \mathbb{E}\left|y_{\Delta}\left(t_{k}\right)\right| \leq \sup _{0 \leq s \leq t} \mathbb{E}\left|y_{\Delta}(s)\right| \\
d\left[y_{\Delta}(t)-\right. & \left.D\left(\bar{y}_{\Delta}(t-\tau), \bar{r}(t)\right)\right] \\
& =F_{\Delta}\left(\bar{y}_{\Delta}(t), \bar{y}_{\Delta}(t-\tau), \bar{r}(t)\right) d t+G_{\Delta}\left(\bar{y}_{\Delta}(t), \bar{y}_{\Delta}(t-\tau), \bar{r}(t)\right) d w(t) .
\end{aligned}
$$




\section{Moment Boundedness of the Truncated EM Method}

In order to obtain moment boundedness of the truncated EM method, we need to show the following lemma.

Lemma 3.1. For any $\Delta \in\left(0, \Delta^{*}\right]$ and let $\hat{p}>0$, we have

$$
\mathbb{E}\left|y_{\Delta}(t)-\bar{y}_{\Delta}(t)\right|^{\hat{p}} \leq C_{\hat{p}} \Delta^{\hat{p} / 2}(\psi(\Delta))^{\hat{p}}, \quad \forall t>0 .
$$

Proof. For all $t>0$ and any $\Delta \in\left(0, \Delta^{*}\right]$, there is a unique integer $k \geq 0$ such that $t_{k} \leq t<t_{k+1}$, we divided the proof into two cases.

Case (I): For $\hat{p} \geq 2$, by (2.14), elementary inequality

$$
|a+b|^{c} \leq 2^{c-1}\left(|a|^{c}+|b|^{c}\right)
$$

for $c>1$, Hölder inequality and Itô isometry, we have

$$
\begin{aligned}
& \mathbb{E}\left|y_{\Delta}(t)-\bar{y}_{\Delta}(t)\right|^{\hat{p}}=\mathbb{E}\left|y_{\Delta}(t)-y_{\Delta}\left(t_{k}\right)\right|^{\hat{p}} \\
\leq & 2^{\hat{p}-1}\left[\mathbb{E}\left|\int_{t_{k}}^{t} F_{\Delta}\left(\bar{y}_{\Delta}(s), \bar{y}_{\Delta}(s-\tau), \bar{r}(s)\right) d s\right|^{\hat{p}}+\mathbb{E}\left|\int_{t_{k}}^{t} G_{\Delta}\left(\bar{y}_{\Delta}(s), \bar{y}_{\Delta}(s-\tau), \bar{r}(s)\right) d w(s)\right|^{\hat{p}}\right] \\
\leq & 2^{\hat{p}-1}\left[\Delta^{p-1} \mathbb{E} \int_{t_{k}}^{t}\left|F_{\Delta}\left(\bar{y}_{\Delta}(s), \bar{y}_{\Delta}(s-\tau), \bar{r}(s)\right)\right|^{\hat{p}} d s\right. \\
& \left.\quad+\Delta^{(\hat{p}-2) / 2} \mathbb{E} \int_{t_{k}}^{t}\left|G_{\Delta}\left(\bar{y}_{\Delta}(s), \bar{y}_{\Delta}(s-\tau), \bar{r}(s)\right)\right|^{\hat{p}} d s\right] \\
\leq & 2^{\hat{p} \Delta^{\hat{p} / 2}(\psi(\Delta))^{\hat{p}}}
\end{aligned}
$$

Case (II): For $\hat{p} \in(0,2)$, by Hölder inequality, we get (3.1).

Now we show the moment boundedness of the truncated EM method.

Lemma 3.2. Assume that $(A 1)-(A 3)$ and $(H 1)$ hold. Then we have

$$
\sup _{0<\Delta \leq \Delta^{*}} \sup _{-\tau \leq t \leq T} \mathbb{E}\left|y_{\Delta}(t)\right|^{p} \leq C
$$

for all $T>0$.

Proof. Fix any $\Delta \in\left(0, \Delta^{*}\right]$ and $0 \leq t \leq T$. Define $z_{\Delta}(t)=y_{\Delta}(t)-D\left(\bar{y}_{\Delta}(t), \bar{r}(t)\right)$. By the Itô formula, we derive from (2.17) that

$$
\begin{aligned}
\mathbb{E}\left|z_{\Delta}(t)\right|^{p} \leq \mathbb{E}\left|z_{\Delta}(0)\right|^{p}+\mathbb{E} \int_{0}^{t} p\left|z_{\Delta}(s)\right|^{p-2}\left(z_{\Delta}^{T}(s) F_{\Delta}\left(\bar{y}_{\Delta}(s), \bar{y}_{\Delta}(s-\tau), \bar{r}(s)\right)\right. \\
\left.\quad+\frac{p-1}{2}\left|G_{\Delta}\left(\bar{y}_{\Delta}(s), \bar{y}_{\Delta}(s-\tau), \bar{r}(s)\right)\right|^{2}\right) d s \\
=:\left|z_{\Delta}(0)\right|^{p}+B_{1}+B_{2}
\end{aligned}
$$

where

$$
\begin{gathered}
B_{1}=\mathbb{E} \int_{0}^{t} p\left|z_{\Delta}(s)\right|^{p-2}\left(\left[\bar{y}_{\Delta}(s)-D\left(\bar{y}_{\Delta}(s-\tau), \bar{r}(s)\right)\right]^{T} F_{\Delta}\left(\bar{y}_{\Delta}(s), \bar{y}_{\Delta}(s-\tau), \bar{r}(s)\right)\right. \\
\left.+\frac{p-1}{2}\left|G_{\Delta}\left(\bar{y}_{\Delta}(s), \bar{y}_{\Delta}(s-\tau), \bar{r}(s)\right)\right|^{2}\right) d s
\end{gathered}
$$


and

$$
B_{2}=\mathbb{E} \int_{0}^{t} p\left|z_{\Delta}(s)\right|^{p-2}\left(y_{\Delta}(s)-\bar{y}_{\Delta}(s)\right)^{T} F_{\Delta}\left(\bar{y}_{\Delta}(s), \bar{y}_{\Delta}(s-\tau), \bar{r}(s)\right) d s .
$$

Using (H1), we get

$$
\begin{gathered}
B_{1} \leq K_{3} \mathbb{E} \int_{0}^{t} p\left|z_{\Delta}(s)\right|^{p-2}\left(1+\left|\bar{y}_{\Delta}(s)\right|^{2}+\left|\bar{y}_{\Delta}(s-\tau)\right|^{2}\right) d s \\
\leq K_{3} p \mathbb{E} \int_{0}^{t}\left|z_{\Delta}(s)\right|^{p-2} d s+K_{3} p \mathbb{E} \int_{0}^{t}\left|z_{\Delta}(s)\right|^{p-2}\left|\bar{y}_{\Delta}(s)\right|^{2} d s \\
+K_{3} p \mathbb{E} \int_{0}^{t}\left|z_{\Delta}(s)\right|^{p-2}\left|\bar{y}_{\Delta}(s-\tau)\right|^{2} d s .
\end{gathered}
$$

By the Young inequality, we compute

$$
\begin{gathered}
p \mathbb{E} \int_{0}^{t}\left|z_{\Delta}(s)\right|^{p-2} d s \leq(p-2) \int_{0}^{t} \mathbb{E}\left|z_{\Delta}(s)\right|^{p} d s+2 T \\
p \mathbb{E} \int_{0}^{t}\left|z_{\Delta}(s)\right|^{p-2}\left|\bar{y}_{\Delta}(s)\right|^{2} d s \leq(p-2) \int_{0}^{t} \mathbb{E}\left|z_{\Delta}(s)\right|^{p} d s+2 \int_{0}^{t} \mathbb{E}\left|\bar{y}_{\Delta}(s)\right|^{p} d s \\
\leq(p-2) \int_{0}^{t} \mathbb{E}\left|z_{\Delta}(s)\right|^{p} d s+2 \int_{0}^{t} \sup _{u \in[0, s]} \mathbb{E}\left|y_{\Delta}(u)\right|^{p} d s \\
p \mathbb{E} \int_{0}^{t}\left|z_{\Delta}(s)\right|^{p-2}\left|\bar{y}_{\Delta}(s-\tau)\right|^{2} d s \\
\leq(p-2) \int_{0}^{t} \mathbb{E}\left|z_{\Delta}(s)\right|^{p} d s+2 \int_{0}^{t} \mathbb{E}\left|\bar{y}_{\Delta}(s-\tau)\right|^{p} d s \\
\leq(p-2) \int_{0}^{t} \mathbb{E}\left|z_{\Delta}(s)\right|^{p} d s+2 \int_{-\tau}^{t} \mathbb{E}\left|\bar{y}_{\Delta}(s)\right|^{p} d s \\
\leq(p-2) \int_{0}^{t} \mathbb{E}\left|z_{\Delta}(s)\right|^{p} d s+2 \tau\|\xi\|^{p}+2 \int_{0}^{t} \sup _{u \in[0, s]} \mathbb{E}\left|y_{\Delta}(u)\right|^{p} d s
\end{gathered}
$$

and

$$
\begin{aligned}
& \mathbb{E} \int_{0}^{t} p\left|z_{\Delta}(s)\right|^{p-2}\left(y_{\Delta}(s)-\bar{y}_{\Delta}(s)\right)^{T} F_{\Delta}\left(\bar{y}_{\Delta}(s), \bar{y}_{\Delta}(s-\tau), \bar{r}(s)\right) d s \\
\leq & (p-2) \mathbb{E} \int_{0}^{t}\left|z_{\Delta}(s)\right|^{p} d s+2 \mathbb{E} \int_{0}^{t}\left|y_{\Delta}(s)-\bar{y}_{\Delta}(s)\right|^{p / 2}\left|F_{\Delta}\left(\bar{y}_{\Delta}(s), \bar{y}_{\Delta}(s-\tau), \bar{r}(s)\right)\right|^{p / 2} d s .
\end{aligned}
$$

By Lemma 3.1, (2.14) and (2.12), we get

$$
\begin{aligned}
& \mathbb{E} \int_{0}^{t}\left|y_{\Delta}(s)-\bar{y}_{\Delta}(s)\right|^{p / 2}\left|F_{\Delta}\left(\bar{y}_{\Delta}(s), \bar{y}_{\Delta}(s-\tau), \bar{r}(s)\right)\right|^{p / 2} d s \\
\leq & (\psi(\Delta))^{p / 2} \mathbb{E} \int_{0}^{t}\left|y_{\Delta}(s)-\bar{y}_{\Delta}(s)\right|^{p / 2} d s \\
\leq & (\psi(\Delta))^{p / 2} \int_{0}^{t} C \Delta^{p / 4}(\psi(\Delta))^{p / 2} d s \leq C .
\end{aligned}
$$

Using elementary inequality

$$
|a+b|^{c} \leq(1+\varsigma)^{c-1}\left(|a|^{c}+\varsigma^{1-c}|b|^{c}\right)
$$


for $c \geq 1, \varsigma>0$ and (A3), we have

$$
\begin{aligned}
\mathbb{E}\left|z_{\Delta}(t)\right|^{p} & \leq(1-\kappa)^{1-p} \mathbb{E}\left|y_{\Delta}(t)\right|^{p}+\kappa^{1-p} E\left|D\left(\bar{y}_{\Delta}(t-\tau), \bar{r}(t)\right)\right|^{p} \\
& \leq(1-\kappa)^{1-p} \mathbb{E}\left|y_{\Delta}(t)\right|^{p}+\kappa \mathbb{E}\left|\bar{y}_{\Delta}(t-\tau)\right|^{p} \\
& \leq(1-\kappa)^{1-p} \mathbb{E}\left|y_{\Delta}(t)\right|^{p}+\kappa \sup _{s \in[0, t]} \mathbb{E}\left|y_{\Delta}(s)\right|^{p}+\kappa\|\xi\|^{p}
\end{aligned}
$$

Hence, we get

$$
\sup _{s \in[0, t]} \mathbb{E}\left|z_{\Delta}(s)\right|^{p} \leq C\left(1+\int_{0}^{t}\left(\sup _{0 \leq u \leq s} \mathbb{E}\left|y_{\Delta}(u)\right|^{p}\right) d s\right) .
$$

By elementary inequality (3.5) and (A3), we obtain

$$
\begin{aligned}
\mathbb{E}\left|y_{\Delta}(t)\right|^{p} & \leq(1-\kappa)^{1-p} \mathbb{E}\left|z_{\Delta}(t)\right|^{p}+\kappa^{1-p} \mathbb{E}\left|D\left(\bar{y}_{\Delta}(t-\tau), \bar{r}(t)\right)\right|^{p} \\
& \leq(1-\kappa)^{1-p} \mathbb{E}\left|z_{\Delta}(t)\right|^{p}+\kappa \sup _{s \in[-\tau, t]} \mathbb{E}\left|y_{\Delta}(s)\right|^{p} \\
& \leq(1-\kappa)^{1-p} \mathbb{E}\left|z_{\Delta}(t)\right|^{p}+\kappa \sup _{s \in[0, t]} \mathbb{E}\left|y_{\Delta}(s)\right|^{p}+\kappa\|\xi\|^{p} .
\end{aligned}
$$

Therefore,

$$
\begin{aligned}
\sup _{s \in[0, t]} \mathbb{E}\left|y_{\Delta}(s)\right|^{p} & \leq \frac{1}{1-\kappa}\left((1-\kappa)^{1-p} \sup _{s \in[0, t]} \mathbb{E}\left|z_{\Delta}(s)\right|^{p}+\kappa\|\xi\|^{p}\right) \\
& \leq C\left(1+\int_{0}^{t}\left(\sup _{0 \leq u \leq s} \mathbb{E}\left|y_{\Delta}(u)\right|^{p}\right) d s\right)
\end{aligned}
$$

where $C$ is independent of $\Delta$.

As this holds for any $t \in[0, T]$ while the right-hand side is non-decreasing in $t$, we can obtain

$$
\sup _{0 \leq u \leq t} \mathbb{E}\left|y_{\Delta}(u)\right|^{p} \leq C\left(1+\int_{0}^{t}\left(\sup _{0 \leq u \leq s} \mathbb{E}\left|y_{\Delta}(u)\right|^{p}\right) d s\right) .
$$

Applying the well-known Gronwall inequality, we obtain

$$
\sup _{0 \leq t \leq T} \mathbb{E}\left|y_{\Delta}(t)\right|^{p} \leq C
$$

It is easy to show

$$
\sup _{-\tau \leq t \leq T} \mathbb{E}\left|y_{\Delta}(t)\right|^{p} \leq C
$$

As this holds for any $\Delta \in\left(0, \Delta^{*}\right]$ while $C$ is independent of $\Delta$, we get the required (3.3). The proof is complete.

Using Lemma 3.2, we can get the following corollaries.

Corollary 3.1. Under $(A 1)-(A 3)$ and $(H 1)$, for any $\Delta \in\left(0, \Delta^{*}\right]$ and $t \in[0, T]$, we have

$$
\mathbb{E}\left|D\left(y_{\Delta}(t-\tau), r(t)\right)-D\left(y_{\Delta}(t-\tau), \bar{r}(t)\right)\right|^{q} \leq C \Delta .
$$

Proof. For all $t \in[0, T]$ and any $\Delta \in\left(0, \Delta^{*}\right]$, by (2.1), (A3) and Lemma 3.2, we have

$$
\begin{aligned}
& \mathbb{E}\left|D\left(y_{\Delta}(t-\tau), r(t)\right)-D\left(y_{\Delta}(t-\tau), \bar{r}(t)\right)\right|^{q} \\
= & \mathbb{E}\left|D\left(y_{\Delta}(t-\tau), r(t)\right)-D\left(y_{\Delta}(t-\tau), r\left(t_{k}\right)\right)\right|^{q}
\end{aligned}
$$




$$
\begin{aligned}
& =\mathbb{E}\left(\left|D\left(y_{\Delta}(t-\tau), r(t)\right)-D\left(y_{\Delta}(t-\tau), r\left(t_{k}\right)\right)\right|^{q} \mathbf{1}_{\left\{r(t) \neq r\left(t_{k}\right)\right\}}\right) \\
& \leq 2^{q-1} \mathbb{E}\left[\left(\left|D\left(y_{\Delta}(t-\tau), r(t)\right)\right|^{q}+\left|D\left(y_{\Delta}(t-\tau), r\left(t_{k}\right)\right)\right|^{q}\right) \mathbf{1}_{\left\{r(t) \neq r\left(t_{k}\right)\right\}}\right] \\
& \leq 2^{q} \kappa^{q} \mathbb{E}\left(\left|y_{\Delta}(t-\tau)\right|^{q} \mathbf{1}_{\left\{r(t) \neq r\left(t_{k}\right)\right\}}\right) \\
& \leq 2^{q} \kappa^{q} \sup _{s \in[-\tau, t]} E\left|y_{\Delta}(s)\right|^{q} E\left(\mathbf{1}_{\left\{r(t) \neq r\left(t_{k}\right)\right\}}\right) \leq C \mathbb{E}\left(\mathbf{1}_{\left\{r(t) \neq r\left(t_{k}\right)\right\}}\right) \\
& =C \sum_{i \in S} \mathbf{1}_{\left\{r\left(t_{k}\right) \neq i\right\}} P\left(r(t) \neq i \mid r\left(t_{k}\right)=i\right) \\
& =C \sum_{i \in S} \mathbf{1}_{\left\{r\left(t_{k}\right) \neq i\right\}} \sum_{j \neq i}\left(\gamma_{i j}\left(t-t_{k}\right)+o\left(t-t_{k}\right)\right) \\
& \leq C \max _{0 \leq i \leq Q}\left(-\gamma_{i i} \Delta+o(\Delta)\right) \sum_{i \in S} \mathbf{1}_{\left\{r\left(t_{k}\right) \neq i\right\}} \\
& \leq C \max _{0 \leq i \leq Q}\left(-\gamma_{i i} \Delta+o(\Delta)\right) \leq C \Delta .
\end{aligned}
$$

This completes the proof of the corollary.

Corollary 3.2. Under $(A 1)-(A 4)$ and $(H 1)$, for any $\Delta \in\left(0, \Delta^{*}\right]$ and $t \in[0, T]$, we have

$$
\begin{aligned}
& \mathbb{E}\left|D(x(t-\tau), r(t))-D\left(\bar{y}_{\Delta}(t-\tau), \bar{r}(t)\right)\right|^{q} \\
\leq & 3^{q-1} \kappa^{q} \sup _{s \in[0, t]} \mathbb{E}\left|x(s)-y_{\Delta}(s)\right|^{q}+C \Delta^{q / 2}(\psi(\Delta))^{q}+C \Delta .
\end{aligned}
$$

Proof. By (A3), Lemma 3.1, Corollary 3.1 and elementary inequality, we have

$$
\begin{aligned}
& \mathbb{E}\left|D(x(t-\tau), r(t))-D\left(\bar{y}_{\Delta}(t-\tau), \bar{r}(t)\right)\right|^{q} \\
\leq & 3^{q-1} \mathbb{E}\left|D(x(t-\tau), r(t))-D\left(y_{\Delta}(t-\tau), r(t)\right)\right|^{q} \\
& +3^{q-1} \mathbb{E}\left|D\left(y_{\Delta}(t-\tau), r(t)\right)-D\left(y_{\Delta}(t-\tau), \bar{r}(t)\right)\right|^{q} \\
& \quad+3^{q-1} \mathbb{E}\left|D\left(y_{\Delta}(t-\tau), \bar{r}(t)\right)-D\left(\bar{y}_{\Delta}(t-\tau), \bar{r}(t)\right)\right|^{q} \\
\leq & 3^{q-1} \kappa^{q} \mathbb{E}\left|x(t-\tau)-y_{\Delta}(t-\tau)\right|^{q}+3^{q-1} C \Delta+3^{q-1} \kappa^{q} \mathbb{E}\left|y_{\Delta}(t-\tau)-\bar{y}_{\Delta}(t-\tau)\right|^{q} \\
\leq & 3^{q-1} \kappa^{q}\left(\sup _{s \in[0, t]} \mathbb{E}\left|x(s)-y_{\Delta}(s)\right|^{q}+\sup _{s \in[-\tau, 0]} \mathbb{E}\left|x(s)-y_{\Delta}(s)\right|^{q}\right) \\
& \quad+3^{q-1} C \Delta+3^{q-1} \kappa^{q} C \Delta^{q / 2}\left(1+(\psi(\Delta))^{q}\right) \\
\leq & 3^{q-1} \kappa^{q} \sup _{s \in[0, t]} \mathbb{E}\left|x(s)-y_{\Delta}(s)\right|^{q}+C \Delta^{q / 2}(\psi(\Delta))^{q}+C \Delta .
\end{aligned}
$$

This completes the proof of the corollary.

\section{Strong Convergence the Truncated EM Method}

\subsection{Strong convergence at time $T$}

Before we show that both truncated EM solutions $y_{\Delta}(T)$ and $\bar{y}_{\Delta}(T)$ will converge to the true solution $x(T)$ in $L^{q}$ for any $T>0$, we need to present several lemmas. In the following of this subsection, we fix $T>0$ arbitrarily.

Lemma 4.1. Let $(A 1)-(A 3)$ hold. For any real number $R>\|\xi\|$, define the stopping time

$$
\sigma_{R}=\inf \{t \geq 0:|x(t)| \geq R\}
$$


where throughout this paper we set $\inf \Phi=\infty$ (and as usual $\Phi$ denotes the empty set). Then

$$
\mathbb{P}\left(\sigma_{R} \leq T\right) \leq \frac{C}{R^{2}},
$$

where $C$ is independent of $\Delta$ and $R$.

Proof. Define $\bar{z}(t)=x(t)-D(x(t-\tau), r(t))$. By the Itô formula and (A4), we derive that

$$
\begin{aligned}
& \mathbb{E}\left|\bar{z}\left(t \wedge \sigma_{R}\right)\right|^{2} \leq \mathbb{E}|\bar{z}(0)|^{2}+\mathbb{E} \int_{0}^{t \wedge \sigma_{R}} 2 K_{1}\left(1+|x(s)|^{2}+|x(s-\tau)|^{2}\right) d s \\
\leq & |\bar{z}(0)|^{2}+2 K_{1} T+2 K_{1} \int_{0}^{t} \mathbb{E}\left|x\left(s \wedge \sigma_{R}\right)\right|^{2} d s+2 K_{1} \int_{0}^{t} \mathbb{E}\left|x\left(s \wedge \sigma_{R}-\tau\right)\right|^{2} d s \\
\leq & |\bar{z}(0)|^{2}+2 K_{1} T+2 K_{1} \int_{0}^{t} \mathbb{E}\left|x\left(s \wedge \sigma_{R}\right)\right|^{2} d s+2 K_{1} \int_{-\tau}^{t} \mathbb{E}\left|x\left(s \wedge \sigma_{R}\right)\right|^{2} d s \\
\leq & |\bar{z}(0)|^{2}+2 K_{1} \tau\|\xi\|^{2}+2 K_{1} T+4 K_{1} \int_{0}^{t} \mathbb{E}\left|x\left(s \wedge \sigma_{R}\right)\right|^{2} d s
\end{aligned}
$$

for any $0 \leq t \leq T$.

Applying elementary equality (3.5) and (A3), we get

$$
|\bar{z}(0)|^{2} \leq 2\left(|\xi(0)|^{2}+\kappa^{2}|\xi(-\tau)|^{2}\right) \leq 2\left(1+\kappa^{2}\right)\|\xi\|^{2},
$$

and

$$
\begin{aligned}
\mathbb{E}|x(t)|^{2} & \leq \frac{1}{\kappa} \mathbb{E}|D(x(t-\tau), r(t))|^{2}+\frac{1}{1-\kappa} \mathbb{E}|\bar{z}(t)|^{2} \\
& \leq \frac{\kappa^{2}}{\kappa} \mathbb{E}|x(t-\tau)|^{2}+\frac{1}{1-\kappa} \mathbb{E}|\bar{z}(t)|^{2} \\
& \leq \kappa \sup _{s \in[-\tau, t]} \mathbb{E}|x(s)|^{2}+\frac{1}{1-\kappa} \mathbb{E}|\bar{z}(t)|^{2} \\
& \leq \kappa\left(\sup _{s \in[0, t]} \mathbb{E}|x(s)|^{2}+\|\xi\|^{2}\right)+\frac{1}{1-\kappa} \mathbb{E}|\bar{z}(t)|^{2} .
\end{aligned}
$$

Hence we have

$$
\begin{aligned}
\sup _{s \in\left[0, t \wedge \sigma_{R}\right]} \mathbb{E}|x(s)|^{2} & \leq \frac{1}{1-\kappa}\left(\frac{1}{1-\kappa} \sup _{s \in\left[0, t \wedge \sigma_{R}\right]} \mathbb{E}|\bar{z}(s)|^{2}+\kappa\|\xi\|^{2}\right) \\
& \leq C\left(1+\int_{0}^{t} \sup _{u \in[0, s]} \mathbb{E}\left|x\left(u \wedge \sigma_{R}\right)\right|^{2} d s\right) .
\end{aligned}
$$

The Gronwall inequality shows

$$
\sup _{s \in[0, T]} \mathbb{E}\left|x\left(s \wedge \sigma_{R}\right)\right|^{2} \leq C,
$$

where $C$ is independent of $\Delta$. This implies

$$
R^{2} \mathbb{P}\left(\sigma_{R} \leq T\right) \leq C
$$

and the assertion follows. 
Lemma 4.2. Let $(A 1)-(A 3)$ and $(H 1)$ hold. For any real number $R>\|\xi\|$ and $\Delta \in\left(0, \Delta^{*}\right]$, define the stopping time

$$
\rho_{\Delta, R}=\inf \left\{t \geq 0:\left|y_{\Delta}(t)\right| \geq R\right\}
$$

Then

$$
\mathbb{P}\left(\rho_{\Delta, R} \leq T\right) \leq \frac{C}{R^{2}}
$$

where $C$ is independent of $\Delta$ and $R$.

Proof. Write $\rho_{\Delta, R}=\rho . z_{\Delta}(t)$ is defined as in the proof of Lemma 3.2. By the Itô formula, we have that for $0 \leq t \leq T$,

$$
\begin{aligned}
& \mathbb{E} \mid z_{\Delta}(t \wedge \rho)\left.\right|^{2} \leq\left|z_{\Delta}(0)\right|^{2}+\mathbb{E} \int_{0}^{t \wedge \rho}\left(2 z_{\Delta}(s)^{T} F_{\Delta}\left(\bar{y}_{\Delta}(s), \bar{y}_{\Delta}(s-\tau), \bar{r}(s)\right)\right. \\
&\left.+\left|G_{\Delta}\left(\bar{y}_{\Delta}(s), \bar{y}_{\Delta}(s-\tau), \bar{r}(s)\right)\right|^{2}\right) d s \\
& \leq\left|z_{\Delta}(0)\right|^{2}+\mathbb{E} \int_{0}^{t \wedge \rho}\left(2\left(\bar{y}_{\Delta}(s)-D\left(\bar{y}_{\Delta}(s-\tau), \bar{r}(s)\right)\right)^{T} F_{\Delta}\left(\bar{y}_{\Delta}(s), \bar{y}_{\Delta}(s-\tau), \bar{r}(s)\right)\right. \\
&\left.+\left|G_{\Delta}\left(\bar{y}_{\Delta}(s), \bar{y}_{\Delta}(s-\tau), \bar{r}(s)\right)\right|^{2}\right) d s \\
&+\mathbb{E} \int_{0}^{t \wedge \rho} 2\left(y_{\Delta}(s)-\bar{y}_{\Delta}(s)\right)^{T} F_{\Delta}\left(\bar{y}_{\Delta}(s), \bar{y}_{\Delta}(s-\tau), \bar{r}(s)\right) d s .
\end{aligned}
$$

By $(\mathrm{H} 1)$, we then obtain that,

$$
\begin{gathered}
\mathbb{E}\left|z_{\Delta}(t \wedge \rho)\right|^{2} \leq\left|z_{\Delta}(0)\right|^{2}+\mathbb{E} \int_{0}^{t \wedge \rho} 2 K_{3}\left(1+\left|\bar{y}_{\Delta}(s)\right|^{2}+\left|\bar{y}_{\Delta}(s-\tau)\right|^{2}\right) d s \\
\left.+\mathbb{E} \int_{0}^{t \wedge \rho} 2 \mid y_{\Delta}(s)-\bar{y}_{\Delta}(s)\right)|| F_{\Delta}\left(\bar{y}_{\Delta}(s), \bar{y}_{\Delta}(s-\tau), \bar{r}(s)\right) \mid d s \\
\leq\left|z_{\Delta}(0)\right|^{2}+2 K_{3} T+4 K_{3} \int_{0}^{t} E\left|\bar{y}_{\Delta}(s \wedge \rho)\right|^{2} d s+2 K_{3} \tau \mathbb{E}\|\xi\|^{2} \\
\left.+2 \mathbb{E} \int_{0}^{T} \mid y_{\Delta}(s)-\bar{y}_{\Delta}(s)\right)|| F_{\Delta}\left(\bar{y}_{\Delta}(s), \bar{y}_{\Delta}(s-\tau), \bar{r}(s)\right) \mid d s \\
\leq\left|z_{\Delta}(0)\right|^{2}+2 K_{3} T+8 K_{3} \int_{0}^{t} \mathbb{E}\left|y_{\Delta}(s \wedge \rho)\right|^{2} d s+8 K_{3} \int_{0}^{T} \mathbb{E}\left|y_{\Delta}(s)-\bar{y}_{\Delta}(s)\right|^{2} d s \\
\left.+2 K_{3} \tau E\|\xi\|^{2}+2 \mathbb{E} \int_{0}^{T} \mid y_{\Delta}(s)-\bar{y}_{\Delta}(s)\right)|| F_{\Delta}\left(\bar{y}_{\Delta}(s), \bar{y}_{\Delta}(s-\tau), \bar{r}(s)\right) \mid d s .
\end{gathered}
$$

Using Corollary 3.1, we have

$$
\left.\int_{0}^{T} \mathbb{E} \mid y_{\Delta}(s)-\bar{y}_{\Delta}(s)\right)\left.\right|^{2} d s \leq T C \Delta(\psi(\Delta))^{2} \leq C T .
$$

While by Lemma 3.2 and (2.14) and (2.12), we derive

$$
\begin{aligned}
& \left.\mathbb{E} \int_{0}^{T} \mid y_{\Delta}(s)-\bar{y}_{\Delta}(s)\right)|| F_{\Delta}\left(\bar{y}_{\Delta}(s), \bar{y}_{\Delta}(s-\tau), \bar{r}(s)\right) \mid d s \\
\leq & \psi(\Delta) \int_{0}^{T} C \Delta^{1 / 2} \psi(\Delta) d s \leq C .
\end{aligned}
$$


Using elementary inequality (3.5) and (A3), we get

$$
\left|z_{\Delta}(0)\right|^{2} \leq 2\left(|\xi(0)|^{2}+\kappa^{2}|\xi(-\tau)|^{2}\right) \leq 2^{p-1}\left(1+\kappa^{2}\right)\|\xi\|^{2},
$$

and

$$
\begin{aligned}
\mathbb{E}\left|y_{\Delta}(t)\right|^{2} & \leq \frac{1}{\kappa} \mathbb{E}\left|D\left(\bar{y}_{\Delta}(t-\tau), \bar{r}(t)\right)\right|^{2}+\frac{1}{1-\kappa} \mathbb{E}\left|z_{\Delta}(t)\right|^{2} \\
& \leq \frac{\kappa^{2}}{\kappa} \mathbb{E}\left|\bar{y}_{\Delta}(t-\tau)\right|^{2}+\frac{1}{1-\kappa} \mathbb{E}\left|z_{\Delta}(t)\right|^{2} \\
& \leq \kappa \sup _{s \in[-\tau, t]} \mathbb{E}\left|\bar{y}_{\Delta}(s)\right|^{2}+\frac{1}{1-\kappa} \mathbb{E}\left|z_{\Delta}(t)\right|^{2} \\
& \leq \kappa\left(\sup _{s \in[0, t]} \mathbb{E}\left|y_{\Delta}(s)\right|^{2}+\|\xi\|^{2}\right)+\frac{1}{1-\kappa} \mathbb{E}\left|z_{\Delta}(t)\right|^{2} .
\end{aligned}
$$

Hence we have

$$
\begin{aligned}
\sup _{s \in\left[0, t \wedge \sigma_{R}\right]} \mathbb{E}\left|y_{\Delta}(s)\right|^{2} & \leq \frac{1}{1-\kappa}\left(\frac{1}{1-\kappa} \sup _{s \in\left[0, t \wedge \sigma_{R}\right]} \mathbb{E}\left|z_{\Delta}(s)\right|^{2}+\kappa\|\xi\|^{2}\right) \\
& \leq C\left(1+\int_{0}^{t} \sup _{u \in[0, s]} \mathbb{E}\left|y_{\Delta}\left(u \wedge \sigma_{R}\right)\right|^{2} d s\right) .
\end{aligned}
$$

The Gronwall inequality shows that

$$
\sup _{s \in[0, T]} \mathbb{E}\left|y_{\Delta}\left(s \wedge \sigma_{R}\right)\right|^{2} \leq C,
$$

where $C$ is independent of $\Delta$ and $R$. This implies the assertion (4.2) easily.

Lemma 4.3. Let $(A 1)-(A 3)$ and $(H 1)$ hold. For any $\Delta \in\left(0, \Delta^{*}\right]$ and $t \in[0, T]$, we have

$$
\begin{aligned}
& \mathbb{E} \int_{0}^{t \wedge \theta_{\Delta, R}}\left|F_{\Delta}\left(y_{\Delta}(s), y_{\Delta}(s-\tau), r(s)\right)-F_{\Delta}\left(y_{\Delta}(s), y_{\Delta}(s-\tau), \bar{r}(s)\right)\right|^{q} d s \leq C(\psi(\Delta))^{q} \Delta, \\
& \mathbb{E} \int_{0}^{t \wedge \theta_{\Delta, R}}\left|G_{\Delta}\left(y_{\Delta}(s), y_{\Delta}(s-\tau), r(s)\right)-G_{\Delta}\left(y_{\Delta}(s), y_{\Delta}(s-\tau), \bar{r}(s)\right)\right|^{q} d s \leq C(\psi(\Delta))^{q} \Delta .
\end{aligned}
$$

Proof. Using elementary inequality, (2.1) and (2.14), we have

$$
\begin{aligned}
& \mathbb{E} \int_{t_{l}}^{t_{l+1}}\left|F_{\Delta}\left(y_{\Delta}(s), y_{\Delta}(s-\tau), r(s)\right)-F_{\Delta}\left(y_{\Delta}(s), y_{\Delta}(s-\tau), \bar{r}(s)\right)\right|^{q} \mathbf{1}_{\left\{r(s) \neq r\left(t_{l}\right)\right\}} d s \\
\leq & 2^{q-1} \mathbb{E} \int_{t_{l}}^{t_{l+1}}\left[\left(\left|F_{\Delta}\left(y_{\Delta}(s), y_{\Delta}(s-\tau), r(s)\right)\right|^{q}\right.\right. \\
& \left.\left.\quad+\left|F_{\Delta}\left(y_{\Delta}(s), y_{\Delta}(s-\tau), r\left(t_{l}\right)\right)\right|^{q}\right) \mathbf{1}_{\left\{r(s) \neq r\left(t_{l}\right)\right\}}\right] d s \\
\leq & 2^{q} \mathbb{E} \int_{t_{l}}^{t_{l+1}}\left((\psi(\Delta))^{q} \mathbf{1}_{\left\{r(s) \neq r\left(t_{l}\right)\right\}}\right) d s \leq C \Delta(\psi(\Delta))^{q} \mathbb{E}\left(\mathbf{1}_{\left\{r(s) \neq r\left(t_{l}\right)\right\}}\right) \\
= & C \Delta(\psi(\Delta))^{q} \sum_{i \in S} \mathbf{1}_{\left\{r\left(t_{l}\right) \neq i\right\}} P\left(r(s) \neq i \mid r\left(t_{l}\right)=i\right) \\
= & C \Delta(\psi(\Delta))^{q} \sum_{i \in S} \mathbf{1}_{\left\{r\left(t_{l}\right) \neq i\right\}} \sum_{j \neq i}\left(\gamma_{i j}\left(s-t_{l}\right)+o\left(s-t_{l}\right)\right)
\end{aligned}
$$




$$
\begin{aligned}
& \leq C \Delta(\psi(\Delta))^{q} \max _{0 \leq i \leq Q}\left(-\gamma_{i i} \Delta+o(\Delta)\right) \sum_{i \in S} \mathbf{1}_{\left\{r\left(t_{l}\right) \neq i\right\}} \\
& \leq C \Delta(\psi(\Delta))^{q} \max _{0 \leq i \leq Q}\left(-\gamma_{i i} \Delta+o(\Delta)\right) \leq C \Delta(\psi(\Delta))^{q}(\Delta+o(\Delta)) .
\end{aligned}
$$

Hence, we get

$$
\begin{aligned}
& \mathbb{E} \int_{0}^{t \wedge \theta_{\Delta, R}}\left|F_{\Delta}\left(y_{\Delta}(s), y_{\Delta}(s-\tau), r(s)\right)-F_{\Delta}\left(y_{\Delta}(s), y_{\Delta}(s-\tau), \bar{r}(s)\right)\right|^{q} d s \\
= & \sum_{l=0}^{\left\lfloor t \wedge \theta_{\Delta, R} / \Delta\right\rfloor} \mathbb{E} \int_{t_{l}}^{t_{l+1}}\left|F_{\Delta}\left(y_{\Delta}(s), y_{\Delta}(s-\tau), r(s)\right)-F_{\Delta}\left(y_{\Delta}(s), y_{\Delta}(s-\tau), r\left(t_{l}\right)\right)\right|^{q} \mathbf{1}_{\left\{r(s) \neq r\left(t_{l}\right)\right\}} d s \\
\leq & C(\psi(\Delta))^{q} \Delta .
\end{aligned}
$$

Similarly, we can obtain the second estimate of the lemma.

In order to show the convergence rate at time $T$, we impose two more assumptions.

(A5) There is a pair of constants $2 \leq q<p$ and $K_{2}>0$ such that

$$
\begin{aligned}
& (x-D(y, i)-\bar{x}+D(\bar{y}, i))^{T}(F(x, y, i)-F(\bar{x}, \bar{y}, i)) \\
& +\frac{3(q-1)}{2}|G(x, y, i)-G(\bar{x}, \bar{y}, i)|^{2} \\
\leq & K_{2}\left(|x-\bar{x}|^{2}+|y-\bar{y}|^{2}\right)
\end{aligned}
$$

for all $x, y, \bar{x}, \bar{y} \in \mathbb{R}^{n}$ and $i \in S$.

(A6) There are constants $K_{4}>0$ and $\gamma>0$ such that

$$
\begin{aligned}
& |F(x, y, i)-F(\bar{x}, \bar{y}, i)| \vee|G(x, y, i)-G(\bar{x}, \bar{y}, i)| \\
\leq & K_{4}\left(1+|x|^{\gamma}+|y|^{\gamma}+|\bar{y}|^{\gamma}+|\bar{x}|^{\gamma}\right)(|x-\bar{x}|+|y-\bar{y}|)
\end{aligned}
$$

for all $x, y, \bar{x}, \bar{y} \in \mathbb{R}^{n}$ and $i \in S$.

Lemma 4.4. Let $(A 2)-(A 6)$ and $(H 1)$ hold. Let $\sigma_{R}$ and $\rho_{\Delta, R}$ be the same as before. Define

$$
\theta_{\Delta, R}:=\sigma_{R} \wedge \rho_{\Delta, R} \text { and } e_{\Delta}(t):=x(t)-y_{\Delta}(t) .
$$

Let $\bar{\Delta} \in\left(0, \Delta^{*}\right]$ be sufficient small such that $\psi(\Delta) \geq \phi(R)$. Then, for any $\Delta \in(0, \bar{\Delta}), q \in[2, p)$ and $q \gamma<p$,

$$
\mathbb{E}\left|e_{\Delta}\left(T \wedge \theta_{\Delta, R}\right)\right|^{q} \leq C \Delta\left[(\psi(\Delta))^{q} \vee 1\right], \quad \forall T>0
$$

Proof. Define $\bar{e}_{\Delta}(t)=x(t)-D(x(t-\tau), r(t))-y_{\Delta}(t)+D\left(\bar{y}_{\Delta}(t-\tau), \bar{r}(t)\right)$. By Itô formula and Young inequality, we can show that for all $t \in[0, T]$,

$$
\begin{aligned}
& \mathbb{E}\left|\bar{e}_{\Delta}\left(t \wedge \theta_{\Delta, R}\right)\right|^{q} \\
& \leq \mathbb{E} \int_{0}^{t \wedge \theta_{\Delta, R}} q\left|\bar{e}_{\Delta}(s)\right|^{q-2}\left(\bar{e}_{\Delta}^{T}(s)\left[F(x(s), x(s-\tau), r(s))-F_{\Delta}\left(\bar{y}_{\Delta}(s), \bar{y}_{\Delta}(s-\tau), \bar{r}(s)\right)\right]\right. \\
&\left.\quad+\frac{q-1}{2}\left|G(x(s), x(s-\tau), r(s))-G_{\Delta}\left(\bar{y}_{\Delta}(s), \bar{y}_{\Delta}(s-\tau), \bar{r}(s)\right)\right|^{2}\right) d s
\end{aligned}
$$




$$
\begin{aligned}
& \leq \mathbb{E} \int_{0}^{t \wedge \theta_{\Delta, R}} q\left|\bar{e}_{\Delta}(s)\right|^{q-2}\left(\overline { e } _ { \Delta } ^ { T } ( s ) \left[\left(F(x(s), x(s-\tau), r(s))-F_{\Delta}\left(y_{\Delta}(s), y_{\Delta}(s-\tau), r(s)\right)\right)\right.\right. \\
& +\left(F_{\Delta}\left(y_{\Delta}(s), y_{\Delta}(s-\tau), r(s)\right)-F_{\Delta}\left(y_{\Delta}(s), y_{\Delta}(s-\tau), \bar{r}(s)\right)\right) \\
& \left.+\left(F_{\Delta}\left(y_{\Delta}(s), y_{\Delta}(s-\tau), \bar{r}(s)\right)-F_{\Delta}\left(\bar{y}_{\Delta}(s), \bar{y}_{\Delta}(s-\tau), \bar{r}(s)\right)\right)\right] \\
& +\frac{3(q-1)}{2}\left[\left|G(x(s), x(s-\tau), r(s))-G_{\Delta}\left(y_{\Delta}(s), y_{\Delta}(s-\tau), r(s)\right)\right|^{2}\right. \\
& +\left|G_{\Delta}\left(y_{\Delta}(s), y_{\Delta}(s-\tau), r(s)\right)-G_{\Delta}\left(y_{\Delta}(s), y_{\Delta}(s-\tau), \bar{r}(s)\right)\right|^{2} \\
& \left.\left.+\left|G\left(y_{\Delta}(s), y_{\Delta}(s-\tau), \bar{r}(s)\right)-G_{\Delta}\left(\bar{y}_{\Delta}(s), \bar{y}_{\Delta}(s-\tau), \bar{r}(s)\right)\right|^{2}\right]\right) d s \\
& \leq \mathbb{E} \int_{0}^{t \wedge \theta_{\Delta, R}} q\left|\bar{e}_{\Delta}(s)\right|^{q-2}\left(\bar{e}_{\Delta}^{T}(s)\left[F(x(s), x(s-\tau), r(s))-F_{\Delta}\left(y_{\Delta}(s), y_{\Delta}(s-\tau), r(s)\right)\right]\right. \\
& \left.+\frac{3(q-1)}{2}\left|G(x(s), x(s-\tau), r(s))-G_{\Delta}\left(y_{\Delta}(s), y_{\Delta}(s-\tau), r(s)\right)\right|^{2}\right) d s \\
& +\mathbb{E} \int_{0}^{t \wedge \theta_{\Delta, R}}(q-2)(3 q-1)\left|\bar{e}_{\Delta}(s)\right|^{q} d s \\
& +\mathbb{E} \int_{0}^{t \wedge \theta_{\Delta, R}} 2\left|\bar{e}_{\Delta}(s)\right|^{q / 2}\left|F_{\Delta}\left(y_{\Delta}(s), y_{\Delta}(s-\tau), r(s)\right)-F_{\Delta}\left(y_{\Delta}(s), y_{\Delta}(s-\tau), \bar{r}(s)\right)\right|^{q / 2} d s \\
& +\mathbb{E} \int_{0}^{t \wedge \theta_{\Delta, R}} 2\left|\bar{e}_{\Delta}(s)\right|^{q / 2}\left|F_{\Delta}\left(y_{\Delta}(s), y_{\Delta}(s-\tau), \bar{r}(s)\right)-F_{\Delta}\left(\bar{y}_{\Delta}(s), \bar{y}_{\Delta}(s-\tau), \bar{r}(s)\right)\right|^{q / 2} d s \\
& +3(q-1) \mathbb{E} \int_{0}^{t \wedge \theta_{\Delta, R}}\left|G\left(y_{\Delta}(s), y_{\Delta}(s-\tau), r(s)\right)-G_{\Delta}\left(y_{\Delta}(s), y_{\Delta}(s-\tau), \bar{r}(s)\right)\right|^{q} d s \\
& +3(q-1) \mathbb{E} \int_{0}^{t \wedge \theta_{\Delta, R}}\left|G_{\Delta}\left(y_{\Delta}(s), y_{\Delta}(s-\tau), \bar{r}(s)\right)-G_{\Delta}\left(\bar{y}_{\Delta}(s), \bar{y}_{\Delta}(s-\tau), \bar{r}(s)\right)\right|^{q} d s \\
& \leq \mathbb{E} \int_{0}^{t \wedge \theta_{\Delta, R}} q\left|\bar{e}_{\Delta}(s)\right|^{q-2}\left(\bar{e}_{\Delta}^{T}(s)\left[F(x(s), x(s-\tau), r(s))-F_{\Delta}\left(y_{\Delta}(s), y_{\Delta}(s-\tau), r(s)\right)\right]\right. \\
& \left.+\frac{3(q-1)}{2}\left|G(x(s), x(s-\tau), r(s))-G_{\Delta}\left(y_{\Delta}(s), y_{\Delta}(s-\tau), r(s)\right)\right|^{2}\right) d s \\
& +\mathbb{E} \int_{0}^{t \wedge \theta_{\Delta, R}}[(q-2)(3 q-1)+2]\left|\bar{e}_{\Delta}(s)\right|^{q} d s \\
& +\mathbb{E} \int_{0}^{t \wedge \theta_{\Delta, R}}\left|F_{\Delta}\left(y_{\Delta}(s), y_{\Delta}(s-\tau), r(s)\right)-F_{\Delta}\left(y_{\Delta}(s), y_{\Delta}(s-\tau), \bar{r}(s)\right)\right|^{q} d s \\
& +\mathbb{E} \int_{0}^{t \wedge \theta_{\Delta, R}}\left|F_{\Delta}\left(y_{\Delta}(s), y_{\Delta}(s-\tau), \bar{r}(s)\right)-F_{\Delta}\left(\bar{y}_{\Delta}(s), \bar{y}_{\Delta}(s-\tau), \bar{r}(s)\right)\right|^{q} d s \\
& +3(q-1) \mathbb{E} \int_{0}^{t \wedge \theta_{\Delta, R}}\left|G_{\Delta}\left(y_{\Delta}(s), y_{\Delta}(s-\tau), r(s)\right)-G_{\Delta}\left(y_{\Delta}(s), y \Delta(s-\tau), \bar{r}(s)\right)\right|^{q} d s \\
& +3(q-1) \mathbb{E} \int_{0}^{t \wedge \theta_{\Delta, R}}\left|G_{\Delta}\left(y_{\Delta}(s), y_{\Delta}(s-\tau), \bar{r}(s)\right)-G_{\Delta}\left(\bar{y}_{\Delta}(s), \bar{y}_{\Delta}(s-\tau), \bar{r}(s)\right)\right|^{q} d s .
\end{aligned}
$$

Using Lemma 4.3, we have

$$
\mathbb{E}\left|\bar{e}_{\Delta}\left(t \wedge \theta_{\Delta, R}\right)\right|^{q}
$$




$$
\begin{array}{rl}
\leq \mathbb{E} \int_{0}^{t \wedge \theta_{\Delta, R}} & q\left|\bar{e}_{\Delta}(s)\right|^{q-2}\left(\bar{e}_{\Delta}^{T}(s)\left[F(x(s), x(s-\tau), r(s))-F_{\Delta}\left(y_{\Delta}(s), y_{\Delta}(s-\tau), r(s)\right)\right]\right. \\
& \left.+\frac{3(q-1)}{2}\left|G(x(s), x(s-\tau), r(s))-G_{\Delta}\left(y_{\Delta}(s), y_{\Delta}(s-\tau), r(s)\right)\right|^{2}\right) d s \\
& +C \mathbb{E} \int_{0}^{t \wedge \theta_{\Delta, R}}\left|\bar{e}_{\Delta}(s)\right|^{q} d s+C(\psi(\Delta))^{q} \Delta \\
& +\mathbb{E} \int_{0}^{t \wedge \theta_{\Delta, R}}\left|F_{\Delta}\left(y_{\Delta}(s), y_{\Delta}(s-\tau), \bar{r}(s)\right)-F_{\Delta}\left(\bar{y}_{\Delta}(s), \bar{y}_{\Delta}(s-\tau), \bar{r}(s)\right)\right|^{q} d s \\
& +3(q-1) \mathbb{E} \int_{0}^{t \wedge \theta_{\Delta, R}}\left|G_{\Delta}\left(y_{\Delta}(s), y_{\Delta}(s-\tau), \bar{r}(s)\right)-G_{\Delta}\left(\bar{y}_{\Delta}(s), \bar{y}_{\Delta}(s-\tau), \bar{r}(s)\right)\right|^{q} d s .
\end{array}
$$

Since $s \in\left[0, t \wedge \theta_{\Delta, R}\right]$

$$
|x(s)| \vee|x(s-\tau)| \vee\left|y_{\Delta}(s)\right| \vee\left|y_{\Delta}(s-\tau)\right| \vee\left|\bar{y}_{\Delta}(s)\right| \vee\left|\bar{y}_{\Delta}(s-\tau)\right| \leq R
$$

and $\psi(\Delta) \geq \phi(R)$, we have

$$
|x(s)| \vee|x(s-\tau)| \vee\left|y_{\Delta}(s)\right| \vee\left|y_{\Delta}(s-\tau)\right| \vee\left|\bar{y}_{\Delta}(s)\right| \vee\left|\bar{y}_{\Delta}(s-\tau)\right| \leq \phi^{-1}(\psi(\Delta))
$$

and

$$
\begin{aligned}
& F_{\Delta}\left(y_{\Delta}(s), y_{\Delta}(s-\tau), r(s)\right)=F\left(y_{\Delta}(s), y_{\Delta}(s-\tau), r(s)\right), \\
& F_{\Delta}\left(y_{\Delta}(s), y_{\Delta}(s-\tau), \bar{r}(s)\right)=F\left(y_{\Delta}(s), y_{\Delta}(s-\tau), \bar{r}(s)\right), \\
& F_{\Delta}\left(\bar{y}_{\Delta}(s), \bar{y}_{\Delta}(s-\tau), \bar{r}(s)\right)=F\left(\bar{y}_{\Delta}(s), \bar{y}_{\Delta}(s-\tau), \bar{r}(s)\right), \\
& G_{\Delta}\left(y_{\Delta}(s), y_{\Delta}(s-\tau), r(s)\right)=G\left(y_{\Delta}(s), y_{\Delta}(s-\tau), r(s)\right), \\
& G_{\Delta}\left(y_{\Delta}(s), y_{\Delta}(s-\tau), \bar{r}(s)\right)=G\left(y_{\Delta}(s), y_{\Delta}(s-\tau), \bar{r}(s)\right), \\
& G_{\Delta}\left(\bar{y}_{\Delta}(s), \bar{y}_{\Delta}(s-\tau), \bar{r}(s)\right)=G\left(\bar{y}_{\Delta}(s), \bar{y}_{\Delta}(s-\tau), \bar{r}(s)\right) .
\end{aligned}
$$

Define $\hat{e}_{\Delta}(t)=x(t)-D(x(t-\tau), r(t))-y_{\Delta}(t)+D\left(y_{\Delta}(t-\tau), \bar{r}(t)\right)$. Consequently, we obtain

$$
\begin{aligned}
& \mathbb{E} \mid \bar{e}_{\Delta}(\left.t \wedge \theta_{\Delta, R}\right)\left.\right|^{q} \\
& \leq \mathbb{E} \int_{0}^{t \wedge \theta_{\Delta, R}} q\left|\bar{e}_{\Delta}(s)\right|^{q-2}\left(\bar{e}_{\Delta}^{T}(s)\left[F(x(s), x(s-\tau), r(s))-F\left(y_{\Delta}(s), y_{\Delta}(s-\tau), r(s)\right)\right]\right. \\
&\left.\quad+\frac{3(q-1)}{2}\left|G(x(s), x(s-\tau), r(s))-G\left(y_{\Delta}(s), y_{\Delta}(s-\tau), r(s)\right)\right|^{2}\right) d s \\
& \quad+C \mathbb{E} \int_{0}^{t \wedge \theta_{\Delta, R}}\left|\bar{e}_{\Delta}(s)\right|^{q} d s+C \Delta(\psi(\Delta))^{q} \\
& \quad+\mathbb{E} \int_{0}^{t \wedge \theta_{\Delta, R}}\left|F\left(y_{\Delta}(s), y_{\Delta}(s-\tau), \bar{r}(s)\right)-F\left(\bar{y}_{\Delta}(s), \bar{y}_{\Delta}(s-\tau), \bar{r}(s)\right)\right|^{q} d s \\
& \quad+3(q-1) \mathbb{E} \int_{0}^{t \wedge \theta_{\Delta, R}}\left|G\left(y_{\Delta}(s), y_{\Delta}(s-\tau), \bar{r}(s)\right)-G\left(\bar{y}_{\Delta}(s), \bar{y}_{\Delta}(s-\tau), \bar{r}(s)\right)\right|^{q} d s \\
& \leq C \mathbb{E} \int_{0}^{t}\left|\bar{e}_{\Delta}\left(s \wedge \theta_{\Delta, R}\right)\right|^{q} d s+C(\psi(\Delta))^{q} \Delta+J_{1}+J_{2}+J_{3}+J_{4},
\end{aligned}
$$

where

$$
J_{1}=\mathbb{E} \int_{0}^{t \wedge \theta_{\Delta, R}} q\left|\bar{e}_{\Delta}(s)\right|^{q-2}\left(\hat{e}_{\Delta}^{T}(s)\left[F(x(s), x(s-\tau), r(s))-F\left(y_{\Delta}(s), y_{\Delta}(s-\tau), r(s)\right)\right]\right.
$$




$$
\begin{aligned}
& \left.+\frac{3(q-1)}{2}\left|G(x(s), x(s-\tau), r(s))-G\left(y_{\Delta}(s), y_{\Delta}(s-\tau), r(s)\right)\right|^{2}\right) d s, \\
J_{2}= & \mathbb{E} \int_{0}^{t \wedge \theta_{\Delta, R}} q\left|\bar{e}_{\Delta}(s)\right|^{q-2}\left(D\left(\bar{y}_{\Delta}(s-\tau), \bar{r}(s)\right)-D\left(y_{\Delta}(s-\tau), \bar{r}(s)\right)\right)^{T} \\
& \cdot\left[F(x(s), x(s-\tau), r(s))-F\left(y_{\Delta}(s), y_{\Delta}(s-\tau), r(s)\right)\right] d s, \\
J_{3}= & \mathbb{E} \int_{0}^{t \wedge \theta_{\Delta, R}}\left|F\left(y_{\Delta}(s), y_{\Delta}(s-\tau), \bar{r}(s)\right)-F\left(\bar{y}_{\Delta}(s), \bar{y}_{\Delta}(s-\tau), \bar{r}(s)\right)\right|^{q} d s, \\
J_{4}= & 3(q-1) \mathbb{E} \int_{0}^{t \wedge \theta_{\Delta, R}}\left|G\left(y_{\Delta}(s), y_{\Delta}(s-\tau), \bar{r}(s)\right)-G\left(\bar{y}_{\Delta}(s), \bar{y}_{\Delta}(s-\tau), \bar{r}(s)\right)\right|^{q} d s .
\end{aligned}
$$

By (A5) and the Young inequality, we get

$$
\begin{aligned}
J_{1} & \leq \mathbb{E} \int_{0}^{t \wedge \theta_{\Delta, R}} q\left|\bar{e}_{\Delta}(s)\right|^{q-2} K_{2}\left(\left|x(s)-y_{\Delta}(s)\right|^{2}+\left|x(s-\tau)-y_{\Delta}(s-\tau)\right|^{2}\right) d s \\
& \leq C\left(\mathbb{E} \int_{0}^{t}\left|\bar{e}_{\Delta}\left(s \wedge \theta_{\Delta, R}\right)\right|^{q} d s+2 \int_{0}^{t} \mathbb{E}\left|e_{\Delta}\left(s \wedge \theta_{\Delta, R}\right)\right|^{q} d s+\int_{-\tau}^{0}\left|\xi\left(\left[\frac{s}{\Delta}\right] \Delta\right)-\xi(s)\right|^{q} d s\right) \\
& \leq C\left(\mathbb{E} \int_{0}^{t}\left|\bar{e}_{\Delta}\left(s \wedge \theta_{\Delta, R}\right)\right|^{q} d s+\int_{0}^{t} \mathbb{E}\left|e_{\Delta}\left(s \wedge \theta_{\Delta, R}\right)\right|^{q} d s+\Delta^{q / 2}\right) .
\end{aligned}
$$

By (A4) and (A6), the Young inequality, Lemma 2.1 and Theorem 3.2, we have that

$$
\begin{aligned}
& J_{2} \leq \mathbb{E} \int_{0}^{t \wedge \theta_{\Delta, R}}\left((q-2)\left|\bar{e}_{\Delta}(s)\right|^{q}+2\left|D\left(\bar{y}_{\Delta}(s-\tau), \bar{r}(s)\right)-D\left(y_{\Delta}(s-\tau), \bar{r}(s)\right)\right|^{q / 2}\right. \\
& \left.\cdot\left|F(x(s), x(s-\tau), r(s))-F\left(\bar{y}_{\Delta}(s), \bar{y}_{\Delta}(s-\tau), r(s)\right)\right|^{q / 2}\right) d s \\
& \leq \mathbb{E} \int_{0}^{t}(q-2)\left|\bar{e}_{\Delta}\left(s \wedge \theta_{\Delta, R}\right)\right|^{q} d s+2\left(K_{4} \kappa\right)^{q / 2} \mathbb{E} \int_{0}^{t \wedge \theta_{\Delta, R}}\left|\bar{y}_{\Delta}(s-\tau)-y_{\Delta}(s-\tau)\right|^{q / 2} \\
& \cdot\left[1+\left|y_{\Delta}(s)\right|^{q \gamma / 2}+|x(s)|^{q \gamma / 2}+\left|y_{\Delta}(s-\tau)\right|^{q \gamma / 2}+|x(s-\tau)|^{q \gamma / 2}\right] \\
& \cdot\left(\left|x(s)-y_{\Delta}(s)\right|^{q / 2}+\left|x(s-\tau)-y_{\Delta}(s-\tau)\right|^{q / 2}\right) d s \\
& \leq \mathbb{E} \int_{0}^{t}(q-2)\left|\bar{e}_{\Delta}\left(s \wedge \theta_{\Delta, R}\right)\right|^{q} d s+C E \int_{0}^{t \wedge \theta_{\Delta, R}}\left|\bar{y}_{\Delta}(s-\tau)-y_{\Delta}(s-\tau)\right|^{q} \\
& \cdot\left[1+\left|y_{\Delta}(s)\right|^{q \gamma}+|x(s)|^{q \gamma}+\left|y_{\Delta}(s-\tau)\right|^{q \gamma}+|x(s-\tau)|^{q \gamma}\right] d s \\
& +C \mathbb{E} \int_{0}^{t \wedge \theta_{\Delta, R}}\left(\left|x(s)-y_{\Delta}(s)\right|^{q}+\left|x(s-\tau)-y_{\Delta}(s-\tau)\right|^{q}\right) d s \\
& \leq \mathbb{E} \int_{0}^{t}(q-2)\left|\bar{e}_{\Delta}\left(s \wedge \theta_{\Delta, R}\right)\right|^{q} d s \\
& +C \int_{0}^{t \wedge \theta_{\Delta, R}}\left(\mathbb{E}\left|\bar{y}_{\Delta}(s-\tau)-y_{\Delta}(s-\tau)\right|^{p q /(p-q \gamma)}\right)^{(p-q \gamma) / p} \\
& \cdot\left[1+\mathbb{E}\left|y_{\Delta}(s)\right|^{p}+\mathbb{E}|x(s)|^{p}+\mathbb{E}\left|y_{\Delta}(s-\tau)\right|^{p}+\mathbb{E}|x(s-\tau)|^{p}\right]^{q \gamma / p} d s \\
& \left.+C \int_{0}^{t} \mathbb{E}\left|e_{\Delta}\left(s \wedge \theta_{\Delta, R}\right)\right|^{q} d s+\int_{-\tau}^{0}\left|\xi\left(\left[\frac{s}{\Delta}\right] \Delta\right)-\xi(s)\right|^{q} d s\right) \\
& \leq C \mathbb{E} \int_{0}^{t}\left|\bar{e}_{\Delta}\left(s \wedge \theta_{\Delta, R}\right)\right|^{q} d s+C \mathbb{E} \int_{0}^{t}\left|e_{\Delta}\left(s \wedge \theta_{\Delta, R}\right)\right|^{q} d s+C \Delta^{q / 2}\left(1+(\psi(\Delta))^{q}\right) \text {. }
\end{aligned}
$$


By Hölder inequality and (A6), we obtain

$$
\begin{aligned}
& J_{3} \leq K_{4}^{q} \mathbb{E} \int_{0}^{t \wedge \theta_{\Delta, R}}\left[1+\left|y_{\Delta}(s)\right|^{q \gamma}+\left|\bar{y}_{\Delta}(s)\right|^{q \gamma}+\left|y_{\Delta}(s-\tau)\right|^{q \gamma}+\left|\bar{y}_{\Delta}(s-\tau)\right|^{q \gamma}\right] \\
& \leq C \int_{0}^{t \wedge \theta_{\Delta, R}}\left[1+\mathbb{E}\left|y_{\Delta}(s)\right|^{p}+\mathbb{E}\left|\bar{y}_{\Delta}(s)\right|^{p}+\mathbb{E}\left|y_{\Delta}(s-\tau)\right|^{p}+\mathbb{E}\left|\bar{y}_{\Delta}(s-\tau)\right|^{p}\right]^{q \gamma / p} \\
& \cdot\left(\mathbb{E}\left|y_{\Delta}(s)-\bar{y}_{\Delta}(s)\right|^{q p /(p-q \gamma)}+\mathbb{E}\left|y_{\Delta}(s-\tau)-\bar{y}_{\Delta}(s-\tau)\right|^{q p /(p-q \gamma)}\right)^{(p-q \gamma) / p} d s \\
& \leq C \Delta^{q / 2}\left(1+(\psi(\Delta))^{q}\right) .
\end{aligned}
$$

Simiarly, we have

$$
J_{4} \leq C \Delta^{q / 2}\left(1+(\psi(\Delta))^{q}\right)
$$

Hence

$$
\mathbb{E}\left|\bar{e}_{\Delta}(t)\right|^{q} \leq C\left(1+\sup _{s \in[0, t]} \mathbb{E}\left|e_{\Delta}(s)\right|^{q}\right)+C \Delta\left[(\psi(\Delta))^{q} \vee 1\right]
$$

Using elementary inequality and Corollary 3.2, we get

$$
\begin{aligned}
\mathbb{E}\left|e_{\Delta}(t)\right|^{q} & \leq 2^{q-1} E\left(\left|\bar{e}_{\Delta}(t)\right|^{q}+\left|D(x(t-\tau), r(t))-D\left(\bar{y}_{\Delta}(t-\tau), \bar{r}(t)\right)\right|^{q}\right) \\
& \leq C\left(1+\sup _{s \in[0, t]} \mathbb{E}\left|\bar{e}_{\Delta}(s)\right|^{q}\right)+C \Delta^{q / 2} .
\end{aligned}
$$

Therefore, we have

$$
\mathbb{E}\left|e_{\Delta}\left(t \wedge \theta_{\Delta, R}\right)\right|^{q} \leq C \int_{0}^{t} \mathbb{E}\left|e_{\Delta}\left(s \wedge \theta_{\Delta, R}\right)\right|^{q} d s+C \Delta\left[(\psi(\Delta))^{q} \vee 1\right]
$$

By Gronwall inequality, we have the assertion (4.5).

Theorem 4.1. Let $(A 2)-(A 6)$ and $(H 1)$ hold. Then, for any $q \in[2, p), p>q \gamma$, and $\psi(\Delta) \geq$ $\phi\left(\Delta\left[(\psi(\Delta))^{q} \vee 1\right]\right)^{-p / 2(p-q)}$, then there is a $\bar{\Delta} \in\left(0, \Delta^{*}\right]$ such that for all $\Delta \in(0, \bar{\Delta}]$

$$
\begin{aligned}
& \mathbb{E}\left|x(T)-y_{\Delta}(T)\right|^{q} \leq C \Delta\left[(\psi(\Delta))^{q} \vee 1\right], \\
& \mathbb{E}\left|x(T)-\bar{y}_{\Delta}(T)\right|^{q} \leq C \Delta\left[(\psi(\Delta))^{q} \vee 1\right] .
\end{aligned}
$$

Proof. Let $\sigma_{R}, \rho_{\Delta, R}, \theta_{\Delta, R}$ and $e_{\Delta}(T)$ be the same as before. By the Young inequality, we obtain that for any $\epsilon>0$,

$$
\begin{gathered}
\mathbb{E}\left|e_{\Delta}(T)\right|^{q}=\mathbb{E}\left(\left|e_{\Delta}(T)\right|^{q} \mathbf{1}_{\left\{\theta_{\Delta, R}>T\right\}}\right)+\mathbb{E}\left(\left|e_{\Delta}(T)\right|^{q} \mathbf{1}_{\left\{\theta_{\Delta, R} \leq T\right\}}\right) \\
\leq \mathbb{E}\left(\left|e_{\Delta}(T)\right|^{q} \mathbf{1}_{\left\{\theta_{\Delta, R}>T\right\}}\right)+\frac{q \epsilon}{p} \mathbb{E}\left|e_{\Delta}(T)\right|^{p}+\frac{p-q}{p \epsilon^{q /(p-q)}} \mathbb{P}\left(\theta_{\Delta, R} \leq T\right) .
\end{gathered}
$$

Using Lemmas 2.1 and 3.2, we have

$$
\mathbb{E}\left|e_{\Delta}(T)\right|^{p} \leq 2^{p-1}\left(\mathbb{E}\left|y_{\Delta}(T)\right|^{p}+\mathbb{E}|y(T)|^{p}\right) \leq C
$$


While by Lemmas 4.1 and 4.2 , we obtain

$$
\mathbb{P}\left(\theta_{\Delta, R} \leq T\right) \leq \mathbb{P}\left(\sigma_{R} \leq T\right)+\mathbb{P}\left(\rho_{\Delta, R} \leq T\right) \leq \frac{C}{R^{2}} .
$$

Substituting (4.10) and (4.11) into (4.9), we hence get

$$
\mathbb{E}\left|e_{\Delta}(T)\right|^{q} \leq \mathbb{E}\left(\left|e_{\Delta}(T)\right|^{q} \mathbf{1}_{\left\{\theta_{\Delta, R}>T\right\}}\right)+\frac{q \epsilon}{p} C+\frac{(p-q) C}{p \epsilon^{q /(p-q)} R^{2}} .
$$

Choosing

$$
\epsilon=\Delta\left[(\psi(\Delta))^{q} \vee 1\right], \quad R=\left(\Delta\left[(\psi(\Delta))^{q} \vee 1\right]\right)^{-p / 2(p-q)},
$$

we have that

$$
\mathbb{E}\left|e_{\Delta}(T)\right|^{q} \leq \mathbb{E}\left(\left|e_{\Delta}(T)\right|{ }^{q} \mathbf{1}_{\left\{\theta_{\Delta, R}>T\right\}}\right)+C \Delta\left[(\psi(\Delta))^{q} \vee 1\right] .
$$

By Lemma 4.4, we can show that

$$
\mathbb{E}\left(\left|e_{\Delta}(T)\right|^{q}\right) \leq C \Delta\left[(\psi(\Delta))^{q} \vee 1\right]
$$

It is easy to see that

$$
\phi^{-1}(\psi(\Delta)) \geq\left(\Delta\left[(\psi(\Delta))^{q} \vee 1\right]\right)^{-p / 2(p-q)}=R .
$$

The proof is therefore complete.

\subsection{Convergence rate over a finite time interval}

We discussed that both truncated EM solutions $y_{\Delta}(T)$ and $\bar{y}_{\Delta}(T)$ converge to the true solution $x(T)$ in $L^{q}$ for any $T>0$ in the previous section. This is sufficient for some applications, but we sometimes need the strong convergence for a numerical solution to the true solution over a finite time interval (see, e.g., [2]). Now we will show the convergence rate over the finite time interval $[0, \mathrm{~T}]$. A stronger assumption is needed.

(A7) There are constants $K>0$ and $\nu>0$ such that

$$
\begin{aligned}
& (x-D(y, i)-\bar{x}+D(\bar{y}, i))^{T}(F(x, y, i)-F(\bar{x}, \bar{y}, i)) \leq K\left(|x-\bar{x}|^{2}+|y-\bar{y}|^{2}\right), \\
& |F(x, y, i)-F(\bar{x}, \bar{y}, i)| \leq K\left(1+|x|^{\nu}+|y|^{\nu}+|\bar{y}|^{\nu}+|\bar{x}|^{\nu}\right)(|x-\bar{x}|+|y-\bar{y}|), \\
& |G(x, y, i)-G(\bar{x}, \bar{y}, i)| \leq K(|x-\bar{x}|+|y-\bar{y}|)
\end{aligned}
$$

for all $x, y, \bar{x}, \bar{y} \in \mathbb{R}^{n}$.

Remark 4.1. We observe that (A7) implies (A1), (A2), (A5) and (A6) we imposed so far in this paper. We now show that (A1), (A2), (A5) and (A6) are satisfied for $p>2$.

$$
\begin{aligned}
& (x-D(y, i))^{T} F(x, y, i) \\
& \quad \leq(0-D(0, i))^{T} F(0,0, i)+(x-D(y, i)-0+D(0, i))^{T}(F(x, y, i)-F(0,0, i)) \\
& \quad \leq K\left(|x|^{2}+|y|^{2}\right) \\
& \quad|G(x, y, i)|^{2} \leq 2|G(0,0, i)|^{2}+2|G(x, y, i)-G(0,0, i)|^{2} \\
& \quad \leq 2|G(0,0, i)|^{2}+4 K^{2}\left(|x|^{2}+|y|^{2}\right) \\
& \quad \leq 2\left(|G(0,0, i)|^{2}+2 K^{2}\right)\left(1+|x|^{2}+|y|^{2}\right),
\end{aligned}
$$




$$
(x-D(y, i))^{T} F(x, y, i)+\frac{p-1}{2}|G(x, y, i)|^{2} \leq K_{1}\left(|x|^{2}+|y|^{2}\right)
$$

and

$$
\begin{aligned}
& (x-D(y, i)-\bar{x}+D(\bar{y}, i))^{T}(F(x, y, i)-F(\bar{x}, \bar{y}, i))+\frac{3(q-1)}{2}|G(x, y, i)-G(\bar{x}, \bar{y}, i)|^{2} \\
\leq & K_{2}\left(|x|^{2}+|y|^{2}\right),
\end{aligned}
$$

where $K_{1}=K+(p-1)\left(|G(0,0, i)|^{2}+2 K^{2}\right)$ and $K_{2}=K+3(q-1) K^{2}$.

Using the same unique as the proof of Lemma 3.2, we therefore have the results under (A7).

Lemma 4.5. Under $(A 3)$ and $(A 7)$, we have

$$
\mathbb{E}\left(\sup _{0 \leq t \leq T}|x(t)|^{p}\right) \leq C .
$$

For any real number $R>\|\xi\|$, define the stopping time

$$
\sigma_{R}=\inf \{t \geq 0:|x(t)| \geq R\}
$$

where throughout this paper we set $\inf \Phi=\infty$ (and as usual $\Phi$ denotes the empty set). Then

$$
\mathbb{P}\left(\sigma_{R} \leq T\right) \leq \frac{C}{R^{p}} .
$$

Proof. $\bar{z}(t)$ is defined se in the proof of Lemma 4.1. Using the Itô formula and (2.17), for $0 \leq t \leq T$, we obtain

$$
\begin{aligned}
|\bar{z}(t)|^{p} \leq & |\bar{z}(0)|^{p}+\int_{0}^{t} p|\bar{z}(s)|^{p-2}\left(\bar{z}^{T}(s) F(x(s), x(s-\tau), r(s))\right. \\
& \left.+\frac{p-1}{2}|G(x(s), x(s-\tau), r(s))|^{2}\right) d s+\int_{0}^{t} p|\bar{z}(s)|^{p-2} \bar{z}^{T}(s) G(x(s), x(s-\tau), r(s)) d w(s) .
\end{aligned}
$$

Therefore we have

$$
\mathbb{E}\left(\sup _{0 \leq u \leq t}|\bar{z}(u)|^{p}\right) \leq|z(0)|^{p}+\bar{M}_{1}+\bar{M}_{2}
$$

where

$$
\begin{aligned}
& \bar{M}_{1}=\mathbb{E} \int_{0}^{t} p|\bar{z}(s)|^{p-2}\left(\bar{z}^{T}(s) F(x(s), x(s-\tau), r(s))+\frac{p-1}{2}|G(x(s), x(s-\tau), r(s))|^{2}\right) d s, \\
& \bar{M}_{2}=\mathbb{E}\left(\left.\sup _{0 \leq u \leq t}\left|\int_{0}^{u} p\right| \bar{z}(s)\right|^{p-2} \bar{z}^{T}(s) G(x(s), x(s-\tau), r(s)) d w(s) \mid\right) .
\end{aligned}
$$

Similarly to the proof of Lemma 3.2, we have

$$
\bar{M}_{1}=C\left(1+\int_{0}^{t}\left(\sup _{0 \leq u \leq s} \mathbb{E}|x(u)|^{p}\right) d s\right) .
$$

Using Young inequality, elementary inequality and Lemma 3.1, we get

$$
\bar{M}_{2} \leq \frac{1}{2} \mathbb{E}\left(\sup _{0 \leq u \leq t}|\bar{z}(u)|^{p}\right)+16 p(p-2) K^{2} \mathbb{E} \int_{0}^{t}|\bar{z}(s)|^{p} d s
$$




$$
\begin{gathered}
+32 p K^{2} \mathbb{E} \int_{0}^{t}\left(1+|x(s)|^{p}+|x(s-\tau)|^{p}\right) d s \\
\leq \frac{1}{2} \mathbb{E}\left(\sup _{0 \leq u \leq t}|\bar{z}(u)|^{p}\right)+C \mathbb{E} \int_{0}^{t}|\bar{z}(s)|^{p} d s+C+C \int_{0}^{t} \mathbb{E}\left(\sup _{0 \leq u \leq s}|x(u)|^{p}\right) d s .
\end{gathered}
$$

Hence, using elementary inequality and (A3), we have

$$
\mathbb{E}\left(\sup _{0 \leq u \leq t}|\bar{z}(u)|^{p}\right) \leq C\left(1+\int_{0}^{t} \mathbb{E}\left(\sup _{0 \leq u \leq s}|x(u)|^{p}\right) d s\right),
$$

where $C$ is independent of $\Delta$ and its value may change between occurrences. By elementary inequality and (A3), we get

$$
\begin{aligned}
\mathbb{E}\left(\sup _{0 \leq u \leq t}|x(u)|^{p}\right) & \leq \frac{C}{1-\kappa}\left(1+\mathbb{E}\left(\sup _{0 \leq u \leq s}|\bar{z}(u)|^{p}\right) d s\right) \\
& \leq C\left(1+\int_{0}^{t} \mathbb{E}\left(\sup _{0 \leq u \leq s}|x(u)|^{p}\right) d s\right) .
\end{aligned}
$$

The well-known Gronwall inequality yields that

$$
\mathbb{E}\left(\sup _{0 \leq t \leq T}|x(t)|^{p}\right) \leq C
$$

Namely,

$$
\mathbb{E}\left(\sup _{-\tau \leq t \leq T}|x(t)|^{p}\right) \leq C .
$$

As this holds for any $\Delta \in\left[0, \Delta^{*}\right]$ while $C$ is independent of $\Delta$. The proof is complete.

Lemma 4.6. Under $(H 1)$, (A3) and $(A 7)$, for any $p>2$, we have

$$
\sup _{0 \leq \Delta \leq \Delta^{*}} \mathbb{E}\left(\sup _{-\tau \leq t \leq T}\left|y_{\Delta}(t)\right|^{p}\right) \leq C .
$$

For any real number $R>\|\xi\|$, define the stopping time

$$
\rho_{\Delta, R}=\inf \left\{t \geq 0:\left|y_{\Delta}(t)\right| \geq R\right\} .
$$

Then

$$
\mathbb{P}\left(\rho_{\Delta, R} \leq T\right) \leq \frac{C}{R^{p}}
$$

Proof. $z_{\Delta}(t)$ is defined se in the proof of Lemma 3.2. By the Itô formula, we derive from (2.17) that, for $0 \leq t \leq T$,

$$
\begin{aligned}
\left|z_{\Delta}(t)\right|^{p} \leq|z(0)|^{p} & +\int_{0}^{t} p\left|z_{\Delta}(s)\right|^{p-2}\left(z_{\Delta}^{T}(s) F_{\Delta}\left(\bar{y}_{\Delta}(s), \bar{y}_{\Delta}(s-\tau), \bar{r}(s)\right)\right. \\
& \left.+\frac{p-1}{2}\left|G_{\Delta}\left(\bar{y}_{\Delta}(s), \bar{y}_{\Delta}(s-\tau), \bar{r}(s)\right)\right|^{2}\right) d s \\
& +\int_{0}^{t} p\left|z_{\Delta}(s)\right|^{p-2} z_{\Delta}^{T}(s) G_{\Delta}\left(\bar{y}_{\Delta}(s), \bar{y}_{\Delta}(s-\tau), \bar{r}(s)\right) d w(s) .
\end{aligned}
$$


Therefore we have

$$
\mathbb{E}\left(\sup _{0 \leq u \leq t}\left|z_{\Delta}(u)\right|^{p}\right) \leq|z(0)|^{p}+M_{1}+M_{2}
$$

where

$$
\begin{aligned}
M_{1}=\mathbb{E} \int_{0}^{t} p\left|z_{\Delta}(s)\right|^{p-2}\left(z_{\Delta}^{T}(s) F_{\Delta}\left(\bar{y}_{\Delta}(s), \bar{y}_{\Delta}(s-\tau), \bar{r}(s)\right)\right. \\
\left.\quad+\frac{p-1}{2}\left|G_{\Delta}\left(\bar{y}_{\Delta}(s), \bar{y}_{\Delta}(s-\tau), \bar{r}(s)\right)\right|^{2}\right) d s \\
M_{2}=\mathbb{E}\left(\left.\sup _{0 \leq u \leq t}\left|\int_{0}^{u} p\right| z_{\Delta}(s)\right|^{p-2} z_{\Delta}^{T}(s) G_{\Delta}\left(\bar{y}_{\Delta}(s), \bar{y}_{\Delta}(s-\tau), \bar{r}(s)\right) d w(s) \mid\right) .
\end{aligned}
$$

Similarly to the proof of Lemma 3.2, we have

$$
M_{1}=C\left(1+\int_{0}^{t}\left(\sup _{0 \leq u \leq s} \mathbb{E}\left|y_{\Delta}(u)\right|^{p}\right) d s\right) .
$$

Using Young inequality, elementary inequality and Lemma 3.1, we get

$$
\begin{aligned}
M_{2} \leq & \frac{1}{2} \mathbb{E}\left(\sup _{0 \leq u \leq t}\left|z_{\Delta}(u)\right|^{p}\right)+16 p(p-2) K^{2} \mathbb{E} \int_{0}^{t}\left|z_{\Delta}(s)\right|^{p} d s \\
& \quad+32 p K^{2} \mathbb{E} \int_{0}^{t}\left(1+\left|\bar{y}_{\Delta}(s)\right|^{p}+\left|\bar{y}_{\Delta}(s-\tau)\right|^{p}\right) d s \\
\leq & \frac{1}{2} \mathbb{E}\left(\sup _{0 \leq u \leq t}\left|z_{\Delta}(t)\right|^{p}\right)+C \mathbb{E} \int_{0}^{t}\left|z_{\Delta}(s)\right|^{p} d s+C \int_{0}^{t} \mathbb{E}\left(\sup _{0 \leq u \leq s}\left|y_{\Delta}(s)\right|^{p}\right) d s+C .
\end{aligned}
$$

Hence, by elementary inequality and (A3), we get

$$
\mathbb{E}\left(\sup _{0 \leq u \leq t}\left|z_{\Delta}(u)\right|^{p}\right) \leq C\left(1+\int_{0}^{t} \mathbb{E}\left(\sup _{0 \leq u \leq s}\left|y_{\Delta}(u)\right|^{p}\right) d s\right),
$$

where $C$ is independent of $\Delta$ and its value may change between occurrences. By elementary inequality and (A3), we have

$$
\begin{aligned}
\mathbb{E}\left(\sup _{0 \leq u \leq t}\left|y_{\Delta}(u)\right|^{p}\right) & \leq \frac{C}{1-\kappa}\left(1+\mathbb{E}\left(\sup _{0 \leq u \leq s}\left|z_{\Delta}(u)\right|^{p}\right) d s\right) \\
& \leq C\left(1+\int_{0}^{t} \mathbb{E}\left(\sup _{0 \leq u \leq s}\left|y_{\Delta}(u)\right|^{p}\right) d s\right) .
\end{aligned}
$$

The well-known Gronwall inequality yields that

$$
\mathbb{E}\left(\sup _{0 \leq t \leq T}\left|y_{\Delta}(t)\right|^{p}\right) \leq C .
$$

Namely,

$$
\mathbb{E}\left(\sup _{-\tau \leq t \leq T}\left|y_{\Delta}(t)\right|^{p}\right) \leq C .
$$

As this holds for any $\Delta \in\left[0, \Delta^{*}\right]$ while $C$ is independent of $\Delta$. The proof is complete. 
Lemma 4.7. Let $(A 3),(A 4),(H 1)$ and $(A 7)$ hold and $q \nu<p$. Let $\bar{\Delta} \in\left(0, \Delta^{*}\right]$ be sufficiently small such that $\psi^{-1}(\phi(\Delta)) \geq R$. Let $\theta_{\Delta, R}, R$ and $e_{\Delta}(t)$ be the same as defined in Theorem 4.1. Let $q \geq 2$ be arbitrary. Then for any $\Delta \in(0, \bar{\Delta})$, we have

$$
\mathbb{E}\left(\sup _{0 \leq t \leq T \wedge \theta_{\Delta, R}}\left|e_{\Delta}(t)\right|^{q}\right) \leq C\left[\Delta(\psi(\Delta))^{q} \vee 1\right] \quad \forall T>0,
$$

where $C$ is dependent on $T, q, \xi$ but independent of $\Delta$.

Proof. We also write $\theta_{\Delta, R}=\theta$ for simplicity. $\bar{e}_{\Delta}(t)$ is defined in the proof of Lemma 4.4. By the Itô formula, we can show that for $0 \leq t \leq T$,

$$
\begin{aligned}
\mathbb{E}\left(\sup _{0 \leq u \leq t \wedge \theta}\left|\bar{e}_{\Delta}(t)\right|^{q}\right) \leq \mathbb{E}\left(\operatorname { s u p } _ { 0 \leq u \leq t \wedge \theta } \int _ { 0 } ^ { u } q | \overline { e } _ { \Delta } ( s ) | ^ { q - 2 } \left[\bar{e}_{\Delta}^{T}(s)[F(x(s), x(s-\tau), r(s))\right.\right. \\
\left.\left.\left.\quad-F_{\Delta}\left(\bar{y}_{\Delta}(s), \bar{y}_{\Delta}(s-\tau), \bar{r}(s)\right)\right]+\frac{q-1}{2}\left|G(x(s), x(s-\tau), r(s))-G_{\Delta}\left(\bar{y}_{\Delta}(s), \bar{y}_{\Delta}(s-\tau), \bar{r}(s)\right)\right|^{2}\right] d s\right) \\
\quad+\mathbb{E}\left(\sup _{0 \leq u \leq t \wedge \theta} \int_{0}^{u} q\left|\bar{e}_{\Delta}(s)\right|^{q-2} \bar{e}_{\Delta}^{T}(s)\left[G(x(s), x(s-\tau), r(s))-G_{\Delta}\left(\bar{y}_{\Delta}(s), \bar{y}_{\Delta}(s-\tau), \bar{r}(s)\right)\right] d w(s)\right) \\
\leq J_{5}+J_{6},
\end{aligned}
$$

where

$$
\begin{aligned}
J_{5}=\mathbb{E}\left(\operatorname { s u p } _ { 0 \leq u \leq t \wedge \theta } \int _ { 0 } ^ { u } q | \overline { e } _ { \Delta } ( s ) | ^ { q - 2 } \left(\bar{e}_{\Delta}(s)^{T}\left[F(x(s), x(s-\tau), r(s))-F_{\Delta}\left(\bar{y}_{\Delta}(s), \bar{y}_{\Delta}(s-\tau), \bar{r}(s)\right)\right]\right.\right. \\
\left.\quad+\frac{3(q-1)}{2}\left|G(x(s), x(s-\tau), r(s))-G_{\Delta}\left(\bar{y}_{\Delta}(s), \bar{y}_{\Delta}(s-\tau), \bar{r}(s)\right)\right|^{2} d s\right) \\
J_{6}=\mathbb{E}\left(\sup _{0 \leq u \leq t \wedge \theta} \int_{0}^{u} q\left|\bar{e}_{\Delta}(s)\right|^{q-2} \bar{e}_{\Delta}^{T}(s)[G(x(s), x(s-\tau), \bar{r}(s))\right. \\
\left.\left.\quad-G_{\Delta}\left(\bar{y}_{\Delta}(s), \bar{y}_{\Delta}(s-\tau), \bar{r}(s)\right)\right] d w(s)\right) .
\end{aligned}
$$

Noting that

$$
\begin{aligned}
& F_{\Delta}\left(y_{\Delta}(s), y_{\Delta}(s-\tau), r(s)\right)=F\left(y_{\Delta}(s), y_{\Delta}(s-\tau), r(s)\right), \\
& F_{\Delta}\left(y_{\Delta}(s), y_{\Delta}(s-\tau), \bar{r}(s)\right)=F\left(y_{\Delta}(s), y_{\Delta}(s-\tau), \bar{r}(s)\right), \\
& F_{\Delta}\left(\bar{y}_{\Delta}(s), \bar{y}_{\Delta}(s-\tau), \bar{r}(s)\right)=F\left(\bar{y}_{\Delta}(s), \bar{y}_{\Delta}(s-\tau), \bar{r}(s)\right), \\
& G_{\Delta}\left(y_{\Delta}(s), y_{\Delta}(s-\tau), r(s)\right)=G\left(y_{\Delta}(s), y_{\Delta}(s-\tau), r(s)\right), \\
& G_{\Delta}\left(y_{\Delta}(s), y_{\Delta}(s-\tau), \bar{r}(s)\right)=G\left(y_{\Delta}(s), y_{\Delta}(s-\tau), \bar{r}(s)\right), \\
& G_{\Delta}\left(\bar{y}_{\Delta}(s), \bar{y}_{\Delta}(s-\tau), \bar{r}(s)\right)=G\left(\bar{y}_{\Delta}(s), \bar{y}_{\Delta}(s-\tau), \bar{r}(s)\right)
\end{aligned}
$$

for $0 \leq s \leq t \wedge \theta$. Similarly to the proof of Lemma 4.4, we can show that

$$
J_{5} \leq C \int_{0}^{t} E\left|e_{\Delta}\left(s \wedge \theta_{\Delta, R}\right)\right|^{q} d s+C\left[\Delta(\psi(\Delta))^{q} \vee 1\right] .
$$


By the Burkholder-Davis-Gundy inequality and (A7), we then have

$$
\begin{aligned}
& J_{6} \leq 4 \sqrt{2} \mathbb{E}\left(\int_{0}^{t \wedge \theta}\left|\bar{e}_{\Delta}(s)^{T}\right|^{2(q-1)}\left|G(x(s), x(s-\tau), \bar{r}(s))-G\left(\bar{y}_{\Delta}(s), \bar{y}_{\Delta}(s-\tau), \bar{r}(s)\right)\right|^{2} d s\right)^{1 / 2} \\
& \leq 12 \sqrt{2} q K \mathbb{E}\left(\operatorname { s u p } _ { 0 \leq u \leq t \wedge \theta } | \overline { e } _ { \Delta } ( u ) ^ { T } | ^ { q } \left[\int_{0}^{t \wedge \theta}\left|\bar{e}_{\Delta}(s)^{T}\right|^{q-2}\right.\right. \\
& \cdot\left[\left|G(x(s), x(s-\tau), r(s))-G_{\Delta}\left(y_{\Delta}(s), y_{\Delta}(s-\tau), r(s)\right)\right|^{2}\right. \\
& +\left|G_{\Delta}\left(y_{\Delta}(s), y_{\Delta}(s-\tau), r(s)\right)-G_{\Delta}\left(y_{\Delta}(s), y_{\Delta}(s-\tau), \bar{r}(s)\right)\right|^{2} \\
& \left.\left.+\left|G\left(y_{\Delta}(s), y_{\Delta}(s-\tau), \bar{r}(s)\right)-G_{\Delta}\left(\bar{y}_{\Delta}(s), \bar{y}_{\Delta}(s-\tau), \bar{r}(s)\right)\right|^{2}\right]^{1 / 2}\right) \\
& \leq \frac{1}{2} \mathbb{E}\left(\sup _{0 \leq u \leq t \wedge \theta}\left|\bar{e}_{\Delta}(u)^{T}\right|^{q}\right)+C \int_{0}^{t} \mathbb{E}\left|\bar{e}_{\Delta}\left(s \wedge \theta_{\Delta, R}\right)\right|^{q} d s \\
& +C \int_{0}^{t} \mathbb{E}\left|e_{\Delta}\left(s \wedge \theta_{\Delta, R}\right)\right|^{q} d s+C\left[\Delta(\psi(\Delta))^{q} \vee 1\right] .
\end{aligned}
$$

Substituting (4.24) and (4.25) into (4.22), we obtain

$$
\mathbb{E}\left(\sup _{0 \leq u \leq t \wedge \theta}\left|\bar{e}_{\Delta}(t)\right|^{q}\right) \leq C\left[\Delta(\psi(\Delta))^{q} \vee 1\right] .
$$

Noting that

$$
\mathbb{E}\left(\sup _{0 \leq u \leq t \wedge \theta}\left|e_{\Delta}(t)\right|^{q}\right) \leq C\left[\Delta(\psi(\Delta))^{q} \vee 1\right]
$$

by (4.8) and the Gronwall inequality, we get (4.21). The proof is complete.

Before we state our theorem in this section, let us remark that it is straightforward to see from (A7) that

$$
\sup _{|x| \vee|y| \leq v}(|F(x, y, i)| \vee|G(x, y, i)|) \leq \bar{K}|v|^{1+\nu}, \quad \forall v \geq 1
$$

where $\bar{K}$ is dependent of $|F(0,0, i)|,|G(0,0, i)|$ and $K$.

Theorem 4.2. Let $(A 3),(A 4),(H 1)$ and $(A 7)$ hold and $\varepsilon \in(0,1 / 2 \wedge 2 / q)$ be arbitrary. Define

$$
\begin{aligned}
& \phi(v)=\bar{K} v^{1+\nu}, \quad v \geq 0, \\
& \psi(\Delta)=\Delta^{-\varepsilon / 2}, \quad \Delta \in(0,1] .
\end{aligned}
$$

Letting $\Delta \in(0, \bar{\Delta})$ be sufficiently small, we can make (2.10), (2.11) and (2.12) hold. Then, for any $q \in[2, p)$ and $q \nu<p$, the truncated EM solutions satisfy

$$
\begin{aligned}
& \mathbb{E}\left(\sup _{0 \leq t \leq T}\left|y_{\Delta}(t)-x(t)\right|^{q}\right)=O\left(\Delta^{1-q \varepsilon / 2}\right), \\
& \mathbb{E}\left(\sup _{0 \leq t \leq T}\left|\bar{y}_{\Delta}(t)-x(t)\right|^{q}\right)=O\left(\Delta^{1-q \varepsilon / 2}\right) .
\end{aligned}
$$


Proof. Let $e_{\Delta}(t)$ and $\theta_{\Delta, R}$ be the same as defined in the proof of Theorem 4.1. Recalling Remark 4.1, we know that the assumptions (A1), (A2), (A5) and (A6) in the previous sections are satisfied under (A7). In particularly, we can choose $p \in[2, p)$ as large as we need for (H1) to hold. For our proof, we choose $p>q \vee(1+\nu)$ sufficiently large such that

$$
\frac{\varepsilon}{2}>\frac{q(1+\nu)}{2(p-q)} .
$$

Using the Young inequality, we can show that, for any $\Delta \in\left(0, \Delta^{*}\right), \epsilon>0$ and $R>\|\xi\|$,

$$
\begin{gathered}
\mathbb{E}\left(\sup _{0 \leq t \leq T}\left|e_{\Delta}(t)\right|^{q}\right) \leq \mathbb{E}\left(\sup _{0 \leq t \leq T}\left|e_{\Delta}(t)\right|^{q} \mathbf{1}_{\left\{\theta_{\Delta, R}>T\right\}}\right)+\frac{q \epsilon}{p} \mathbb{E}\left(\sup _{0 \leq t \leq T}\left|e_{\Delta}(t)\right|^{p}\right) \\
+\frac{p-q}{p \epsilon^{q /(p-q)}} \mathbb{P}\left(\theta_{\Delta, R} \leq T\right) .
\end{gathered}
$$

By Lemmas 4.5, 4.1 and 4.2, we can then have

$$
\mathbb{E}\left(\sup _{0 \leq t \leq T}\left|e_{\Delta}(t)\right|^{q}\right) \leq \mathbb{E}\left(\sup _{0 \leq t \leq T}\left|e_{\Delta}(t)\right|^{q} \mathbf{1}_{\left\{\theta_{\Delta, R}>T\right\}}\right)+\frac{q \epsilon}{p} C+\frac{C(p-q)}{p \epsilon^{q /(p-q)} R^{p}} .
$$

We therefore see that the inequality

$$
\mathbb{E}\left(\sup _{0 \leq t \leq T}\left|e_{\Delta}(t)\right|^{q}\right) \leq \mathbb{E}\left(\sup _{0 \leq t \leq T}\left|e_{\Delta}\left(t \wedge \theta_{\Delta, R}\right)\right|^{q}\right)+\frac{q \epsilon}{p} C+\frac{C(p-q)}{p \epsilon^{q /(p-q)} R^{p}}
$$

holds for any $\Delta \in(0, \bar{\Delta}), \epsilon>0$. Choosing $\epsilon=\Delta^{1-q \varepsilon / 2}$ and $R=\left(\Delta^{1-q \varepsilon / 2}\right)^{-1 /(p-q)}$, we then get

$$
\mathbb{E}\left(\sup _{0 \leq t \leq T}\left|e_{\Delta}(t)\right|^{q}\right) \leq \mathbb{E}\left(\sup _{0 \leq t \leq T}\left|e_{\Delta}\left(t \wedge \theta_{\Delta, R}\right)\right|^{q}\right)+C \Delta^{1-q \varepsilon / 2}
$$

for any $\Delta \in(0, \bar{\Delta})$. On the other hand, by (4.29), we see that

$$
\Delta^{-\varepsilon / 2} \geq \bar{K}\left(\Delta^{q(1-\varepsilon)(1+\nu) / 2} \vee \Delta^{(1+\nu)}\right)^{-1 /(p-q)}
$$

for all sufficiently small $\Delta$. For every such small $\Delta$, we then have

$$
\phi^{-1}(\psi(\Delta)) \geq\left(\Delta^{1-q \varepsilon / 2}\right)^{-1 /(p-q)}=R .
$$

By Lemma 4.7, we have

$$
\mathbb{E}\left(\sup _{0 \leq t \leq T}\left|e_{\Delta}(t)\right|^{q}\right) \leq C\left(\Delta^{1-q \varepsilon / 2}\right)
$$

for any $\Delta \in(0, \bar{\Delta})$. In other words, the required (4.27) has been proved. By the similar way of Lemma 5.4 in [17], we have

$$
\mathbb{E}\left(\sup _{0 \leq t \leq T}\left|y_{\Delta}(t)-\bar{y}_{\Delta}(t)\right|^{q}\right) \leq C\left(\Delta^{q(1-\varepsilon) / 2}\right)
$$

for any $\Delta \in(0, \bar{\Delta})$. This, together with (4.34), implies

$$
\mathbb{E}\left(\sup _{0 \leq t \leq T}\left|\bar{y}_{\Delta}(t)-x(t)\right|^{q}\right) \leq\left(\Delta^{1-q \varepsilon / 2}\right)
$$

for all sufficiently small $\Delta$, we must therefore have (4.28) as desired. 


\section{Numerical Experiments}

In this section, to illustrate the therotical results, we present two numerical examples.

Example 5.1. Consider the NSDDE-MS

$$
\begin{gathered}
d(x(t)-0.1 x(t-1))=a(r(t))\left[x(t)-0.1 x(t-1)-(x(t)-0.1 x(t-1))^{3}\right] d t \\
+b(r(t))|x(t)-0.1 x(t-1)|^{3 / 2} d w(t)
\end{gathered}
$$

on $t \geq 0$ with initial data $\{y(t)=1:-\tau \leq t \leq 0\}$. where $w(t)$ is a 1-dimension Brownian motion, $r(t)$ is a Markovian chain on the state space $S=\{1,2\}$ and they are independent. Let the generator of Markovain chain that

$$
\Gamma=\left(\begin{array}{rc}
-1 & 1 \\
2 & -2
\end{array}\right) .
$$

Moreover, $a(1)=2, b(1)=1, a(2)=1, b(2)=2$.

Here $D(y, i)=0.1 y, F(x, y, i)=a(r(t))\left[x-0.1 y-(x-0.1 y)^{3}\right]$ and $G(x, y, i)=b(r(t)) \mid x-$ $\left.0.1 y\right|^{3 / 2}$. It is easy to see that (A1), (A3) and (A4) are satisfied. It can be seen that

$$
\begin{aligned}
& (x-D(y, i))^{T} F(x, y, i)+\frac{q-1}{2}|G(x, y, i)|^{2} \\
= & (x-0.1 y)^{T} 2\left[x-0.1 y-(x-0.1 y)^{3}\right]+\frac{q-1}{2}|| x-\left.\left.0.1 y\right|^{3 / 2}\right|^{2} \\
= & (x-0.1 y)^{2}\left[2-2(x-0.1 y)^{2}+\frac{q-1}{2}|x-0.1 y|\right] \\
\leq & (x-0.1 y)^{2}\left[2+\frac{(q-1)^{2}}{32}\right] \\
\leq & 2\left[2+\frac{(q-1)^{2}}{32}\right]\left(|x|^{2}+|y|^{2}\right)
\end{aligned}
$$

and

$$
\begin{aligned}
& (x-D(y, i))^{T} F(x, y, i)+\frac{q-1}{2}|G(x, y, i)|^{2} \\
= & (x-0.1 y)^{T}\left[x-0.1 y-(x-0.1 y)^{3}\right]+\frac{q-1}{2}|2| x-\left.\left.0.1 y\right|^{3 / 2}\right|^{2} \\
= & (x-0.1 y)^{2}\left[1-(x-0.1 y)^{2}+2(q-1)|x-0.1 y|\right] \\
\leq & (x-0.1 y)^{2}\left[1+(q-1)^{2}\right] \\
\leq & 2\left[1+(q-1)^{2}\right]\left(|x|^{2}+|y|^{2}\right) .
\end{aligned}
$$

Hence, if we choose $K_{1}=2\left[2+\frac{(q-1)^{2}}{32}\right] \vee\left[1+(q-1)^{2}\right]$, we have (A2). Obviously,

$$
\begin{aligned}
& |F(x, y, i)-F(\bar{x}, \bar{y}, i)| \\
= & |a(i)|\left|x-0.1 y-(x-0.1 y)^{3}-(\bar{x}-0.1 \bar{y})+(\bar{x}-0.1 \bar{y})^{3}\right| \\
\leq & 2\left|1-(x-0.1 y)^{2}-(\bar{x}-0.1 \bar{y})^{2}-(x-0.1 y)(\bar{x}-0.1 \bar{y})\right|(|x-\bar{x}|+0.1|y-\bar{y}|) \\
\leq & 2\left|1-\frac{1}{2}(x-0.1 y)^{2}-\frac{1}{2}(\bar{x}-0.1 \bar{y})^{2}\right|(|x-\bar{x}|+0.1|y-\bar{y}|)
\end{aligned}
$$




$$
\begin{aligned}
& \leq 2\left(1+|x|^{2}+0.01|y|^{2}+|\bar{x}|^{2}+0.01|\bar{y}|^{2}\right)(|x-\bar{x}|+0.1|y-\bar{y}|) \\
& \leq 2\left(1+|x|^{2}+|y|^{2}+|\bar{x}|^{2}+|\bar{y}|^{2}\right)(|x-\bar{x}|+0.1|y-\bar{y}|)
\end{aligned}
$$

and

$$
\begin{aligned}
& |G(x, y, i)-G(\bar{x}, \bar{y}, i)|=|b(i)||| x-\left.0.1 y\right|^{3 / 2}-|\bar{x}-0.1 \bar{y}|^{3 / 2} \mid \\
\leq & 2|| x-\left.0.1 y\right|^{1 / 2}-|\bar{x}-0.1 \bar{y}|^{1 / 2} \mid\left(|x-0.1 y|+|\bar{x}-0.1 \bar{y}|+|x-0.1 y|^{1 / 2}|\bar{x}-0.1 \bar{y}|^{1 / 2}\right) \\
\leq & 2|| x-\left.0.1 y\right|^{1 / 2}-|\bar{x}-0.1 \bar{y}|^{1 / 2} \mid\left(|x-0.1 y|^{1 / 2}+|\bar{x}-0.1 \bar{y}|^{1 / 2}\right)^{2} \\
\leq & 2|| x-0.1 y|-| \bar{x}-0.1 \bar{y}||\left(|x-0.1 y|^{1 / 2}+|\bar{x}-0.1 \bar{y}|^{1 / 2}\right) \\
\leq & 2\left(|x|^{1 / 2}+|y|^{1 / 2}+|\bar{x}|^{1 / 2}+|\bar{y}|^{1 / 2}\right)|x-0.1 y-\bar{x}+0.1 \bar{y}| \\
\leq & 2\left(|x|^{1 / 2}+|y|^{1 / 2}+|\bar{x}|^{1 / 2}+|\bar{y}|^{1 / 2}\right)(|x-\bar{x}|+|y-\bar{y}|) .
\end{aligned}
$$

Therefore, (A6) is satisfied with $\gamma=2$.

$$
\begin{aligned}
& {[x-D(y, i)-\bar{x}-D(\bar{y}, i)]^{T}[F(x, y, i)-F(\bar{x}, \bar{y}, i)]+\frac{3(q-1)}{2}|G(x, y, i)-G(\bar{x}, \bar{y}, i)|^{2} } \\
= & {[x-0.1 y-(\bar{x}-0.1 \bar{y})]^{T} 2\left[x-0.1 y-(x-0.1 y)^{3}-(\bar{x}-0.1 \bar{y})+(\bar{x}-0.1 \bar{y})^{3}\right] } \\
& \quad+\frac{3(q-1)}{2} \| x-\left.0.1 y\right|^{3 / 2}-\left.|\bar{x}-0.1 \bar{y}|^{3 / 2}\right|^{2} \\
\leq & {[x-0.1 y-(\bar{x}-0.1 \bar{y})]^{2}\left[2-\left((x-0.1 y)^{2}+(\bar{x}-0.1 \bar{y})^{2}\right)\right] } \\
& \quad+\frac{3(q-1)}{2}|| x-0.1 y|-| \bar{x}-\left.0.1 \bar{y}\right|^{2}\left(|x-0.1 y|^{1 / 2}+|\bar{x}-0.1 \bar{y}|^{1 / 2}\right)^{2} \\
\leq & {[x-0.1 y-(\bar{x}-0.1 \bar{y})]^{2}\left[2-\left((x-0.1 y)^{2}+(\bar{x}-0.1 \bar{y})^{2}\right)+3(q-1)(|x-0.1 y|+|\bar{x}-0.1 \bar{y}|)\right] } \\
\leq & 2\left(2+\frac{9}{2}(q-1)^{2}\right)\left(|x-\bar{x}|^{2}+|y-\bar{y}|^{2}\right)
\end{aligned}
$$

and

$$
\begin{aligned}
& {[x-D(y, i)-\bar{x}-D(\bar{y}, i)]^{T}[F(x, y, i)-F(\bar{x}, \bar{y}, i)]+\frac{3(q-1)}{2}|G(x, y, i)-G(\bar{x}, \bar{y}, i)|^{2} } \\
= & {[x-0.1 y-(\bar{x}-0.1 \bar{y})]^{T}\left[x-0.1 y-(x-0.1 y)^{3}-(\bar{x}-0.1 \bar{y})+(\bar{x}-0.1 \bar{y})^{3}\right] } \\
& \quad+\frac{3(q-1)}{2}|2| x-\left.0.1 y\right|^{3 / 2}-\left.2|\bar{x}-0.1 \bar{y}|^{3 / 2}\right|^{2} \\
\leq & {[x-0.1 y-(\bar{x}-0.1 \bar{y})]^{2}\left[1-\frac{1}{2}\left((x-0.1 y)^{2}+(\bar{x}-0.1 \bar{y})^{2}\right)\right] } \\
& \quad+6(q-1)|| x-0.1 y|-| \bar{x}-\left.0.1 \bar{y}\right|^{2} \mid\left(|x-0.1 y|^{1 / 2}+|\bar{x}-0.1 \bar{y}|^{1 / 2}\right)^{2} \\
\leq & {[x-0.1 y-(\bar{x}-0.1 \bar{y})]^{2}\left[1-\frac{1}{2}\left((x-0.1 y)^{2}+(\bar{x}-0.1 \bar{y})^{2}\right)\right.} \\
& \quad+6(q-1)(|x-0.1 y|+|\bar{x}-0.1 \bar{y}|)] \\
\leq & 2\left(1+36(q-1)^{2}\right)\left(|x-\bar{x}|^{2}+|y-\bar{y}|^{2}\right) .
\end{aligned}
$$

So (A2) is also fulfilled.

Then we define the truncated EM functions. Let $\phi(s)=2 s^{3}$ such that

$$
\sup _{|x| \vee|y| \leq s}(|F(x, y, i)| \vee|F(x, y, i)|)=\sup _{|x| \vee|y| \leq s}\left(2|x|^{3} \vee 2|x|^{3 / 2}\right) \leq 2 s^{3}, \quad \forall s \geq 1
$$


Let $\psi(\Delta)=\Delta^{-1 / 10}$, then $(2.10)-(2.12)$ hold for any $\Delta^{*} \in(0,1]$. Now we define the truncated functions as follows:

$$
\begin{aligned}
& F_{\Delta}(x, y, i)=F\left(\left(|x| \wedge \Delta^{-1 / 30}\right) \frac{x}{|x|},\left(|y| \wedge \Delta^{-1 / 30}\right) \frac{y}{|y|}, i\right), \\
& G_{\Delta}(x, y, i)=G\left(\left(|x| \wedge \Delta^{-1 / 30}\right) \frac{x}{|x|},\left(|y| \wedge \Delta^{-1 / 30}\right) \frac{y}{|y|}, i\right) .
\end{aligned}
$$

We use discrete Brownian paths over $[0,1]$ with $\Delta=2^{-12}$. We take the numerical solution with $h=\Delta$ to be a approximation $X_{\Delta}$ of the exact solution and compare this with the numerical approximation using $h=2^{6} \Delta, h=2^{7} \Delta, h=2^{8} \Delta$ and $h=2^{9} \Delta$ over $M=500$ sample paths. Here the mean-square error is denoted as follows:

$$
\text { Error }_{h}:=\left(\frac{1}{M} \sum_{i=1}^{M}\left|Y_{h}^{i}(T)-X_{\Delta}^{i}(T)\right|^{2}\right)^{1 / 2}
$$

where $Y_{h}^{i}(T)$ denotes the numerical solution of the truncated EM method along the $i$ th sample path at $t=T$ with stepsize $h$, and the strong convergence order is defined numerically by

$$
\text { Order }=\log \frac{\text { Error }_{h}}{\text { Error }_{h / 2}} / \log (2) .
$$

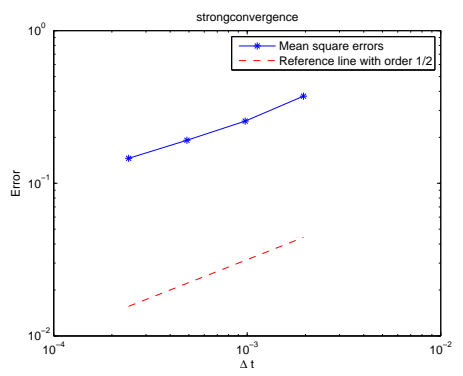

Fig. 5.1. Strong convergence of the truncated EM for NSDDE-MS (5.1).

The strong convergence order of truncated EM for NSDDE-MS (5.1) is shown in Fig. 5.1. From this, we can see that the strong convergence order of truncated EM for NSDDE-MS (5.1) is close to $\frac{1}{2}$.

Example 5.2. Consider the NSDDE-MS

$$
d(x(t)-0.1 \sin (x(t-1)))=\left[-a(r(t))\left(x(t)^{3}\right] d t+x d w(t),\right.
$$

on $t \geq 0$ with initial data $\{y(t)=1:-\tau \leq t \leq 0\}$. where $w(t)$ is a 1-dimension Brownian motion, $r(t)$ is a Markovian chain on the state space $S=\{1,2\}$ and they are independent. Let the generator of Markovain chain that

$$
\Gamma=\left(\begin{array}{rc}
-1 & 1 \\
2 & -2
\end{array}\right)
$$

Moreover, $a(1)=2, a(2)=1$. 
Here $D(y, i)=0.1 \sin y, F(x, y, i)=-a(r(t)) x^{3}$ and $G(x, y, i)=x$. It is obvious that (A1), (A3) and (A4) are satisfied. It can be seen that

$$
\begin{aligned}
& (x-D(y, i))^{T} F(x, y, i)+\frac{q-1}{2}|G(x, y, i)|^{2} \\
& =(x-0.1 \sin y)^{T} 2\left[-x^{3}\right]+\frac{q-1}{2}|x|^{2} \\
& =x^{2}\left[-2 x^{2}+\frac{q-1}{2}\right] \leq \frac{q-1}{2}|x|^{2}, \\
& (x-D(y, i))^{T} F(x, y, i)+\frac{q-1}{2}|G(x, y, i)|^{2} \leq \frac{q-1}{2}|x|^{2} .
\end{aligned}
$$

Hence, if we choose $K_{1}=\frac{q-1}{2}$, we have (A2). Obviously,

$$
\begin{aligned}
& |F(x, y, i)-F(\bar{x}, \bar{y}, i)|=|a(i)|\left|-x^{3}+\bar{x}^{3}\right| \\
\leq & 2\left(|x|^{2}+|\bar{x}|^{2}+|x||\bar{x}|\right)|x-\bar{x}| \leq 3\left(|x|^{2}+|\bar{x}|^{2}\right)|x-\bar{x}|, \\
& |G(x, y, i)-G(\bar{x}, \bar{y}, i)|=|x-\bar{x}| .
\end{aligned}
$$

Therefore, (A7) is satisfied.

$$
\begin{aligned}
& {[x-D(y, i)-\bar{x}-D(\bar{y}, i)]^{T}[F(x, y, i)-F(\bar{x}, \bar{y}, i)] } \\
= & {[x-0.1 \sin y-(\bar{x}-0.1 \sin \bar{y})]^{T} 2\left[-x^{3}+\bar{x}^{3}\right] a(r(t)) } \\
\leq & \left.|x-\bar{x}|^{2}\left[-x^{2}-\bar{x}^{2}-x \bar{x}\right)\right] a(r(t))+|-0.1 \sin y+0.1 \sin \bar{y}|\left|-x^{3}+\bar{x}^{3}\right| a(r(t)) \\
\leq & \left.|x-\bar{x}|^{2}\left[-x^{2}-\bar{x}^{2}-x \bar{x}\right)\right] a(r(t))+0.2\left|x-\bar{x} \| x^{2}+\bar{x}^{2}+x \bar{x}\right| a(r(t)) \\
\leq & \left.|x-\bar{x}|^{2}\left[-x^{2}-\bar{x}^{2}-x \bar{x}\right)\right] a(r(t))+0.2\left|x-\bar{x} \|(x-\bar{x})^{2}+3 x \bar{x}\right| a(r(t)) \\
\leq & \left.|x-\bar{x}|^{2}\left[-x^{2}-\bar{x}^{2}-x \bar{x}\right)\right] a(r(t))+0.2\left|x-\bar{x} \|(x-\bar{x})^{2}+\frac{3}{2}(x-\bar{x})^{2}\right| a(r(t)) \\
\leq & \left.|x-\bar{x}|^{2}\left[-x^{2}-\bar{x}^{2}-x \bar{x}+0.5|x-\bar{x}|\right)\right] a(r(t)) \\
\leq & \left.|x-\bar{x}|^{2}\left[-0.5 x^{2}-0.5 \bar{x}^{2}+0.5|x|+0.5|\bar{x}|\right)\right] a(r(t)) \\
\leq & \frac{1}{2}|x-\bar{x}|^{2} .
\end{aligned}
$$

So (A7) is also fulfilled.

Then we define the truncated EM functions. Let $\phi(s)=2 s^{3}$ such that

$$
\sup _{|x| \vee|y| \leq s}(|F(x, y, i)| \vee|G(x, y, i)|)=\sup _{|x| \vee|y| \leq s}\left(|x|^{3} \vee|x|\right) \leq 2 s^{3}, \quad \forall s \geq 1
$$

Let $\psi(\Delta)=\Delta^{-1 / 10}$, then $(2.10)-(2.12)$ hold for any $\Delta^{*} \in(0,1]$. Now we define the truncated functions as follows:

$$
\begin{aligned}
& F_{\Delta}(x, y, i)=F\left(\left(|x| \wedge \Delta^{-1 / 30}\right) \frac{x}{|x|},\left(|y| \wedge \Delta^{-1 / 30}\right) \frac{y}{|y|}, i\right), \\
& G_{\Delta}(x, y, i)=G\left(\left(|x| \wedge \Delta^{-1 / 30}\right) \frac{x}{|x|},\left(|y| \wedge \Delta^{-1 / 30}\right) \frac{y}{|y|}, i\right) .
\end{aligned}
$$

Consider NSDDE-MS (5.4) with $T=1$ and $\Delta=2^{-12}$. We use the numerical solution with $h=\Delta$ to be a approximation $\tilde{Y}_{\Delta}$ of the exact solution and compare this with the numerical 
approximation using $h=2^{6} \Delta, h=2^{7} \Delta, h=2^{8} \Delta$ and $h=2^{9} \Delta$ over $M=1000$ sample paths. Here the mean-square error is denoted as follows:

$$
\text { Error }_{h}:=\left(\frac{1}{M} \sum_{j=1}^{M}\left|Y_{h}^{j}(T)-\tilde{Y}_{\Delta}^{j}(T)\right|^{2}\right)^{1 / 2}
$$

where $Y_{h}^{i}(T)$ denotes the numerical solution of the truncated EM method along the $i$ th sample path at $t=T$ with stepsize $h$, and the strong convergence order is defined numerically by

$$
\text { Order }=\log \frac{\text { Error }_{h}}{\text { Error }_{h / 2}} / \log (2) .
$$

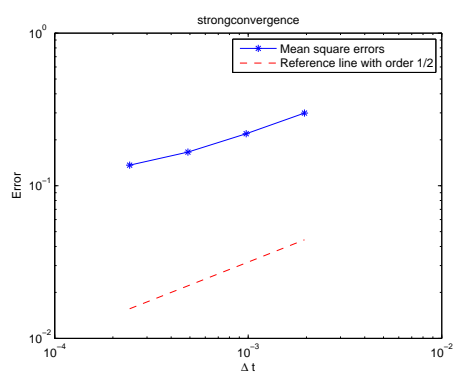

Fig. 5.2. Strong convergence of the truncated EM for NSDDE-MS (5.4).

The strong convergence order of truncated EM for NSDDE-MS (5.4) is shown in Fig. 5.2. From this, we can see that the strong convergence order of truncated EM for NSDDE-MS (5.4) is close to $\frac{1}{2}$.

\section{Conclusion}

This paper aims to present an explicit method, called the truncated EM method, for the nonlinear NSDDEs-MS. The moment boundedness is investigated. We also show strong convergence rates at time $T$ and over a finite interval $[0, T]$.

\section{References}

[1] Q. Guo, X. Mao, R. Yue, The truncated Euler-Maruyama method for stochastic differential delay equations, Numer. Algorithms, 78:2 (2018), 599-624.

[2] I. Gyöngy, A note on Eulers approximations, Potential Anal., 8:3 (1998), 205-216.

[3] L. Hu, X. Li, X. Mao, Convergence rate and stability of the truncated Euler-Maruyama method for stochastic differential equations, J. Comput. Appl. Math., 337 (2018), 274-289.

[4] M. Hutzethaler, A. Jentzen, and P. E. Kloeden, Strong and weak divergence in finite time of Eulers method for stochastic differential equations with non-globally Lipschitz continuous coefficients, $P$. Roy. Soc. A, 467 (2010), 1563-1576.

[5] M. Hutzenthaler, A. Jentzen, P.E. Kloeden, Strong convergence of an explicit numerical method for SDEs with nonglobally Lipschitz continuous coefficients, Ann. Appl. Probab., 22:4 (2012), 1611-1641.

[6] I. Ya. Kac, N.N. Krasovskii, About stability of systems with stochastic parameters, Prikladnaya Matematika i Mekhanika, 24 (1960), 809-823 (in Russian). 
[7] I. Ya. Kac, Method of Lyapunov functions in problems of stability and stabilization of systems of stochastic structure, Ekaterinburg, 1998 (in Russian).

[8] V. Kolmanovskii, N. Koroleva, T. Maizenberg, et al., Neutral stochastic differential delay equations with Markovian switching, Stoch. Anal. Appl., 21:4 (2003), 819-847.

[9] B. Li, D. Xu, Attraction of stochastic neutral delay differential equations with Markovian switching, IMA J. Math. Control I., 31:1 (2013), 15-31.

[10] X. Li, X. Mao, A note on almost sure asymptotic stability of neutral stochastic delay differential equations with Markovian switching, Automatica, 48:9 (2012), 2329-2334.

[11] R. Li, Y.H. Dai, H.B. Meng, Convergence of numerical solutions to stochastic differential equations with Markovian switching, Appl. Math. Comput., 315:4 (2017), 744-752.

[12] X. Li, X. Mao, G. Yin, Explicit numerical approximations for stochastic differential equations in finite and infinite horizons: truncation methods, convergence in pth moment, and stability, IMA J. Numer. Anal., 2018.

[13] X. Mao, C. Yuan, Stochastic differential equations with Markovian switching, London, U.K. Imperial College Press, 2006.

[14] X. Mao, Stochastic differential equations and applications(second edition), Horwood Pub Limited, 1997.

[15] X. Mao, A. Matasov, A.B. Piunovskiy, Stochastic differential delay equations with Markovian switching, Bernoulli. 6:1 (2000), 73-90.

[16] X. Mao, M.J. Rassias, Khasminskii-type theorems for stochastic differential delay equations, Stoch. Anal. Appl., 23 (2005), 1045-1069.

[17] X. Mao, The truncated Euler-maruyama method for stochastic differential equations, J. Comput. Appl. Math., (2015), 370-384.

[18] X. Mao, Convergence rates of the truncated Euler-maruyama method for stochastic differential equations, J. Comput. Appl. Math., 296 (2016), 362-375.

[19] Son L. Nguyen, et al., Milstein-Type procedures for numerical solutions of stochastic differential equations with Markovian switching, SIAM J. Numer. Anal., 55:2 (2017), 953-979.

[20] Dung T. Nguyen, et al., Tamed-Euler method for hybrid stochastic differential equations with Markovian switching, Nonlinear Anal. Hybrid Systems, 30 (2018), 14-30.

[21] R.F. Pawula, N.J. Bershad, Random differential equations in science and engineering, New York: Academic Press, 1973.

[22] Y. Xu, Z. He, Exponential stability of neutral stochastic delay differential equations with Markovian switching, Appl. Math. Lett., 52 (2016), 64-73.

[23] H. Yang, F. Jiang, Approximations of numerical method for neutral stochastic functional differential equations with Markovian switching, J. Appl. Math., (2012), 991-1073.

[24] B. Yin, and Z. Ma, Convergence of the semi-implicit Euler method for neutral stochastic delay differential equations with phase semi-Markovian switching, Appl. Math. Model., 35:5 (2011), 2094-2109.

[25] C. Yuan, X. Mao, Convergence of the Euler-Maruyama method for stochastic differential equations with Markovian switching, Math. Comput. Simulat., 64:2 (2004), 223-235.

[26] C. Yuan, Numerical solutions and stability of stochastic differential equations with Markovian switching, J. Comput. Appl. Math., 174:1 (2004), 1-27.

[27] C. Yuan, W. Glover, Approximate solutions of stochastic differential delay equations with Markovian switching, J. Differ. Equ. Appl., 16:2-3 (2010), 195-207.

[28] W. Zhang, M. Song, M. Liu, Strong Convergence of the Partially Truncated Euler-Maruyama Method for a Class of Stochastic Differential Delay Equations, J. Comput. Appl. Math., 335 (2018), 114-128.

[29] W. Zhang, M. Song, M. Liu, Strong Convergence of the truncated Euler-Maruyama method of stochastic functional differential equations, Intertional Journal of Computer Mathematics, 95:12 (2018), 2363-2387.

[30] S. Zhou, F. Wu, Convergence of numerical solutions to neutral stochastic delay differential equations with Markovian switching, J. Comput. Appl. Math., 229 (2009), 85-96.

[31] E. Zhu, X. Tian, Y. Wang, On $p$ th moment exponential stability of stochastic differential equations with Markovian switching and time-varying delay, J. Inequal. Appl., (2015), 1-11. 